\title{
Synthesis of 5-nitro-pyrazole triflones via [3+2] cycloaddition reaction and its application for potential insecticide
}

\author{
Pulakesh Das, ${ }^{1}$ Hiroto Uno, ${ }^{1}$ Etsuko Tokunaga,${ }^{1}$ Yuji Sumii ${ }^{1}$ and Norio Shibata ${ }^{1,2 *}$ \\ ${ }^{1}$ Department of Nanopharmaceutical Sciences, and Department of Life Science and Applied Chemistry, \\ Nagoya Institute of Technology, Gokiso, Showa-ku, Nagoya 466-8555, Japan \\ E-mail: nozshiba@nitech.ac.jp; TEL; +81-52-735-7543 \\ ${ }^{2}$ Institute of Advanced Fluorine-Containing Materials, Zhejiang Normal University, 688 Yingbin Avenue, 321004 \\ Jinhua, China \\ Abstract: Synthesis of 5-nitro-pyrazole triflones 6 was achieved via [3+2] cycloaddition reaction of 2-diazo-1- \\ phenyl-2-((trifluoromethyl)sulfonyl)ethan-1-one (3) and $\alpha$-bromonitrostyrene derivatives 7 under basic \\ conditions in moderate to good yields. An agrochemically attractive 5-amino-N-pyrimidinyl-pyrazole triflone $2 a$ \\ was successfully prepared from 5-nitro-pyrazole triflone $6 c$ in two steps.
}

Key words: 5-nitro-pyrazole triflones, $\alpha$-bromonitrostyrene, [3+2] cycloaddition

Introduction: Pyrazoles are often found as an integral part of biologically active molecules [1]. In particular, pyrazoles with aryl or heteroaryl substituents at $\mathrm{N}-1$ position and a free $\mathrm{NH}_{2}$ group at $\mathrm{C}-5$ position have gathered much attention because of their promising pharmaceutical [2] and agrochemical [3] properties. More precisely, 1-arylpyrazoles having substituents like alkyl, thioalkyl, acyl or cyano- group at the C-3 and C-4 positions exhibit potent insecticidal activities [4]. Amongst the pyrazole-type pesticides, Fipronil ${ }^{\circledR}$ (5-amino-1(2,6-dichloro-4-trifluoromethylphenyl)-4-trifluoromethanesulfinyl-1H-pyrazole-3-carbonitrile) is a highly effective broad-spectrum insecticide and veterinary medicine to get rid of fleas and ticks [5] (Figure 1). However, the use of Fipronil ${ }^{\circledR}$ is the matter of discussion due to the recent food safety incident [6]. Thus, the development of safer derivatives of Fipronil ${ }^{\circledR}$ is of great importance. Bayer CropScience AG has filed patents on the sulfurderivatized pyrimidinyl-pyrazol-5-amines 1 which showed potent activities as insecticides and/or parasiticides $[3 a, 7]$. Inspired by their patents, we are interested in triflyl (trifluoromethanesulfonyl, $\mathrm{SO}_{2} \mathrm{CF}_{3}$ ) variants (triflones) $\mathbf{2}$ as potential pesticides.

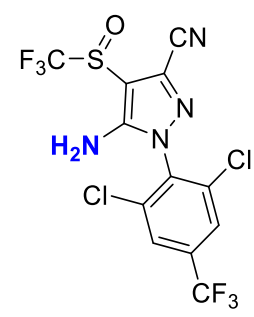

Fipronil $^{\circledR}$

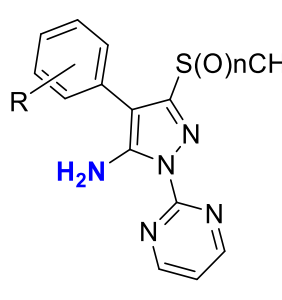

1

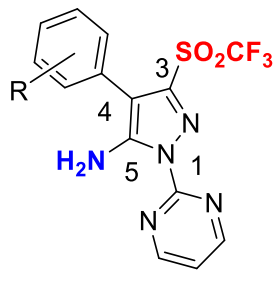

2

Figure 1. Fipronil ${ }^{\oplus}$ and its biologically attractive derivatives containing aryl or heteroaryl substituent at $N-1$ position and a free $\mathrm{NH}_{2}$ group at $C-5$ position. 
The introduction of fluorine(s) and fluorinated functional groups into organic compounds is one of the effective strategies to improve/alter biological activity and metabolic stability of original molecules [8]. In the case of our target 5-amino-pyrazole triflones $\mathbf{2}$, the incorporation of a lipophilic triflyl group $(\pi=0.55)$ to $C$-3 position in $\mathbf{2}$ could potentially perturb the chemical, physical and biological properties of the parent heterocyclic 5-amino-pyrazoles. Besides, the triflone variants $\mathbf{2}$ are also expected to have a reduced basicity of the amine moiety in 2 due to the strong electron withdrawing effect by the triflyl group $\left(\sigma_{\mathrm{m}}=0.79, \sigma_{\mathrm{p}}=0.93\right)$ [9]. For decades, our group has been focusing on the development of novel shelf-stable reagents for fluorofunctionalization reactions [10,11]. One such kind of unique compounds is 2-diazo-1-phenyl-2((trifluoromethyl)-sulfonyl)ethan-1-one (3) [12]. The compound $\mathbf{3}$ was originally developed by us as a regent for electrophilic trifluoromethylthiolation reactions. A wide variety of nucleophiles such as enamines, indoles, $\beta$ ketoesters, and pyrroles are nicely trifluoromethylthiolated by $\mathbf{3}$ in the presence of a copper catalyst under mild conditions [12a]. Aromatic coupling reaction is also available by using $\mathbf{3}$. On the other hand, $\mathbf{3}$ also acts as a powerful building block for the preparation of variety of triflones such as $\beta$-lactam triflones [12c]. Recently, we have reported the synthesis of pyrazole triflones $\mathbf{4}$ via [3+2] cycloaddition of $\mathbf{3}$ with nitrostyrenes $\mathbf{5}$ (Scheme 1a) [12d]. In the presence of $\mathrm{NaOMe}$, reagent $\mathbf{3}$ generates a reactive anionic triflyl-diazomethane species $\mathbf{A}$ in situ to react with nitrostyrenes 5. As an extension of our research work on heterocyclic triflones [12], we herein report the novel strategy for the preparation of 5-amino- $N$-pyrimidinyl-pyrazole triflones 2 . The important precursors to access $\mathbf{2}$ are the 5-nitro-pyrazole triflones $\mathbf{6}$, which can be synthesized by the reaction of $\mathbf{3}$ with $\alpha$ bromonitrostyrenes 7 under basic conditions (Scheme 1b). The selective elimination of the bromo group rather than nitro group in $\mathbf{7}$ during the cyclization step to suppress the formation of 5-bromo-pyrazole triflones $\mathbf{8}$ is the key for the success [13]. Substrate scope of $\mathbf{7}$ for the preparation of $\mathbf{6}$ was examined. Subsequently, the 5-nitropyrazole triflone $6 \mathrm{c}$ was successfully transformed to the targeted 5 -amino- $N$-pyrimidinyl-pyrazole triflone $\mathbf{2 a}$ via two steps in moderated yield (Scheme 1b).

a) previous work

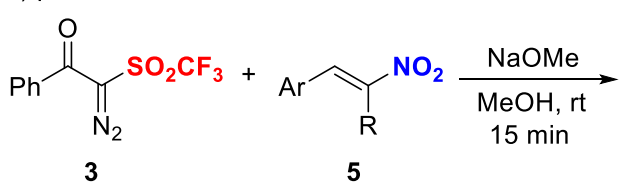

b) this work<smiles>CO[N+](=O)[O-]</smiles>

3
7

$\mathrm{Cl}$<smiles>C=C[CH]Cl</smiles><smiles>[R]c1[nH]nc(S(=O)(=O)OC(F)(F)F)c1[Te]</smiles>

4

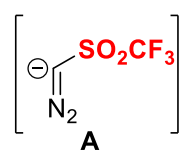

anionic triflyl-diazomethane

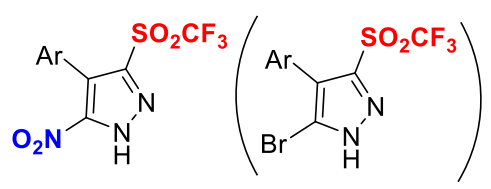

6

8

Ar: $4-\mathrm{Cl}_{-} \mathrm{C}_{6} \mathrm{H}_{4}$<smiles>Nc1c(-c2ccccc2)c(S(=O)(=O)OC(F)(F)F)nn1-c1ncccn1</smiles>

Scheme 1. a) Synthesis of pyrazole triflons $\mathbf{4}$ via [3+2] cycloaddition of $\mathbf{3}$ with nitrostyrenes $\mathbf{5}$ (previous work); b) Selective synthesis of 5-nitro-pyrazole triflons 6 and transformation of 6 c to 5 -amino- $N$-pyrimidinyl-pyrazole triflone $\mathbf{2 a}$ (this work). 
Based on our previous work [12d], we initiated to optimize the reaction temperatures for the reactions between 3 and $\alpha$-bromonitrostyrene 7a in the presence of 10 equiv of $\mathrm{NaOMe}$ in $\mathrm{MeOH}$ (Table 1). Desired 5nitro-pyrazole triflone 6 a was obtained in $62 \%$ yield along with 5 -bromo-pyrazole triflone 8 a in $17 \%$ yield at $0{ }^{\circ} \mathrm{C}$ for $20 \mathrm{~min}$ (entry 1). While, decreasing the reaction temperature to $-10{ }^{\circ} \mathrm{C}$, did not affect the yield and products distribution (entry 2). Increasing the amount of 3 from 1.2 to 1.5 equiv, slightly increased the yields of 6 a to $64 \%$ and $8 \mathrm{a}$ to $19 \%$ (entry 3 ) were detected, whereas lowering the temperature with 1.5 equiv of 3 did not affect the formation of nitro product $6 a$ with slightly increased the yield of bromo 8 a to $21 \%$ (entry 4). Thus, the combination of 3 (1.5 equiv) and $\alpha$-bromonitrostyrenes 7 (1.0 equiv) in the presence of NaOMe (10.0 equiv) in $\mathrm{MeOH}$ at $0{ }^{\circ} \mathrm{C}$ for $20 \mathrm{~min}$, was selected as the optimized reaction condition (entry 3 ).

Table 1. Optimization of $[3+2]$ cycloaddition reaction ${ }^{a}$

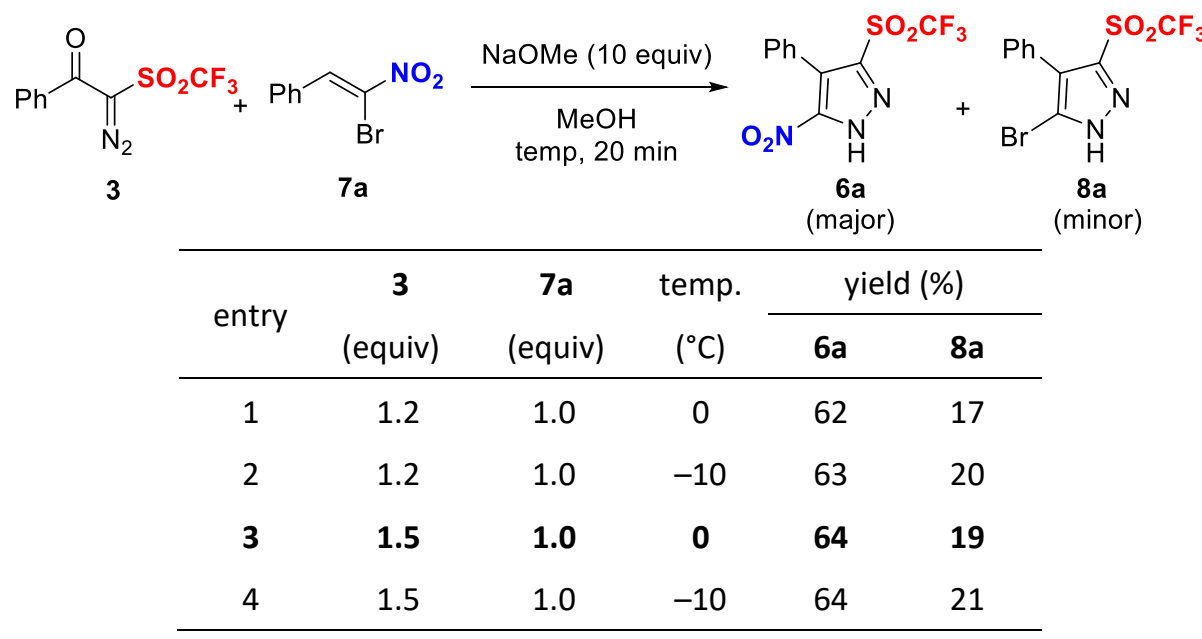

${ }^{a}$ Reaction conditions: Experiments were performed with 3 (1.2 or 1.5 equiv), 7a (1.0 equiv), NaOMe (10 equiv), $\mathrm{MeOH}(1.0 \mathrm{~mL})$ at given temperature for $20 \mathrm{~min}$.

With the optimized reaction conditions in hand, the substrate scope of the $[3+2]$ cyclization reaction was explored as shown in Table 2. All the reactions proceeded smoothly to furnish the desired 5-nitro-pyrazole triflones $\mathbf{6 b}-\mathbf{e}$ as major products in good yields with similar selectivity as 7a. Electron donating 4- $\mathrm{Me}-\mathrm{C}_{6} \mathrm{H}_{4}$ substituted substrate $\mathbf{7 b}$ provided the product in similar yield and selectivity (6b: $67 \%, \mathbf{8 b}: \mathbf{2 3} \%$ ). The halogen substituted $\alpha$-bromonitrostyrenes $\left(7 \mathrm{c}: 4-\mathrm{Cl}-\mathrm{C}_{6} \mathrm{H}_{4} ; 7 \mathrm{~d}\right.$ : 3- $\left.\mathrm{Br}-\mathrm{C}_{6} \mathrm{H}_{4} ; 7 \mathrm{e}: 4-\mathrm{F}-\mathrm{C}_{6} \mathrm{H}_{4}\right)$ provided the corresponding 5nitro-pyrazole triflones 6 with higher yields (6c: 77\%; 6d: 76\%; 6e: 69\%) along with corresponding bromo byproducts 8 (8c: $15 \%$; 8 d: $18 \%$; 8 e: $17 \%$ ) (Table 2$)$.

Next, we examined the synthesis of 5 -amino- $N$-pyrimidinyl-pyrazole triflone $2 \mathbf{a}$. The reduction of nitro group in $\mathbf{6 c}$ by hydrogenation in the presence of $\mathrm{Pd}-\mathrm{C}$ in $\mathrm{MeOH}$ gave the 5-amino-pyrazole triflone $\mathbf{9}$, followed by the nucleophilic addition to 2-chloropyrimidine in the presence of $\mathrm{NaH}$ under heating condition to afford the potential biological active 5-amino- $N$-pyrimidinyl-pyrazole triflone 2 a in $35 \%$ yield for two steps (Scheme 2). 
Table 2. Scope of the reaction of diazotriflone $\mathbf{3}$ with $\alpha$-bromonitroalkenes $7 a-e^{a}$

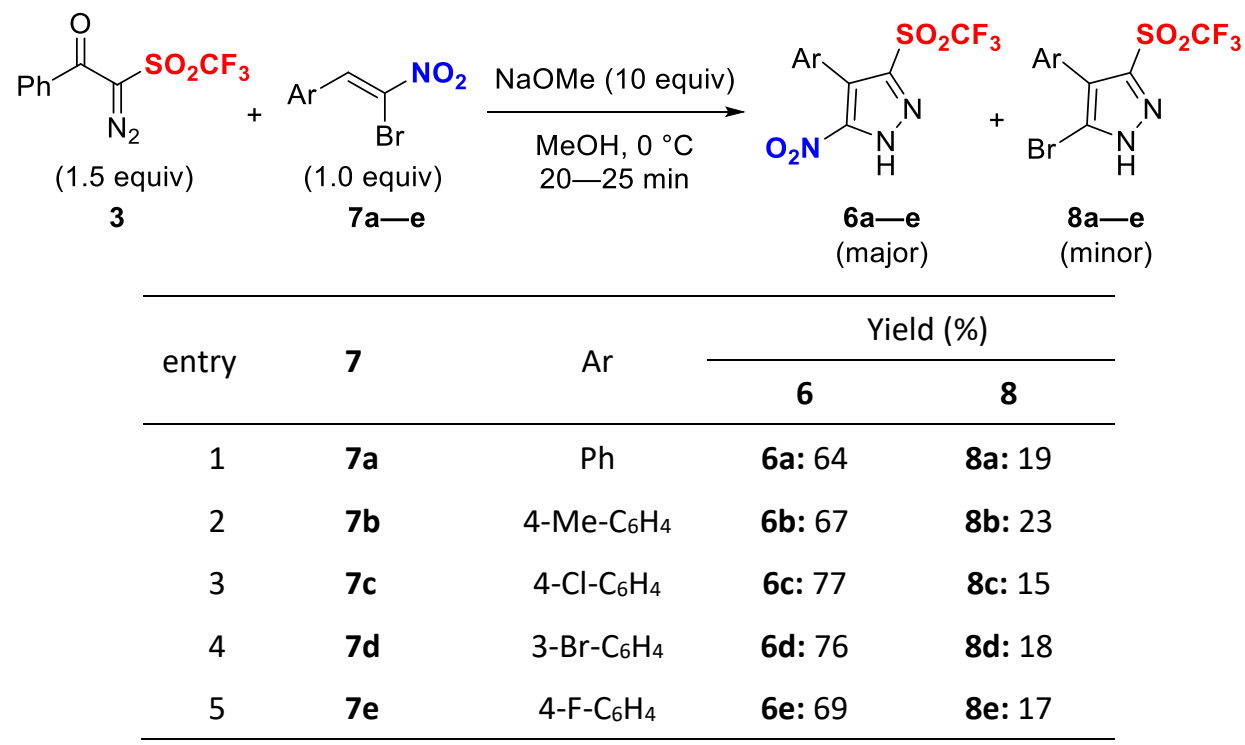

${ }^{a}$ Experiments were performed with 3 (1.5 equiv), 7a-e (1.0 equiv) and $\mathrm{NaOMe}\left(10\right.$ equiv) in dry $\mathrm{MeOH}$ at $0{ }^{\circ} \mathrm{C}$ for 20-25 min.

Scheme 2. Synthesis of 5-amino- $N$-pyrimidinyl-pyrazole triflone $\mathbf{2 a}$.

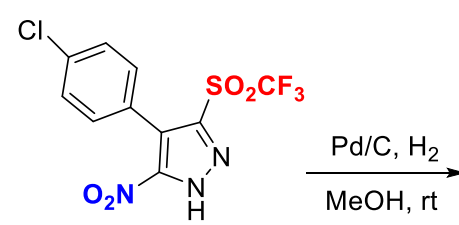

$6 c$<smiles>Nc1[nH]nc(S(=O)(=O)F)c1-c1ccc(Cl)cc1</smiles>

9

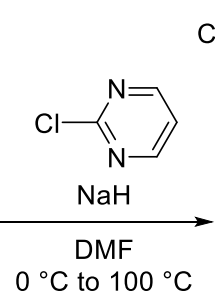

$35 \%$ in 2 steps

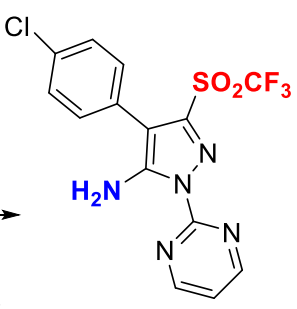

$2 \mathbf{a}$

In conclusion, we have disclosed the synthesis of 5-nitro-pyrazole triflones 6 via [3+2] cycloaddition reaction of 2-diazo-1-phenyl-2-((trifluoromethyl)sulfonyl)ethan-1-one $\mathbf{3}$ and $\alpha$-bromonitrostyrene derivatives 7 under basic conditions in moderate to good yields. The transformation of 5-nitro-pyrazole triflone 6 to an agrochemically attractive 5-amino- $N$-pyrimidinyl-pyrazole triflone 2 a was successfully demonstrated in two steps. The synthesis of a series of 5-amino- $N$-pyrimidinyl-pyrazole triflones and their evaluation of the insecticide property are underway.

\section{Experimental}

All reactions were performed in oven-dried glassware under positive pressure of nitrogen or argon unless mentioned otherwise. Solvents were transferred via syringe and were introduced into reaction vessels though a rubber septum. All reactions were monitored by thin-layer chromatography (TLC) carried out on $0.25 \mathrm{~mm}$ Merck silica gel $\left(60-\mathrm{F}_{254}\right)$. The TLC plates were visualized with UV light and $\mathrm{KMnO}_{4}$ in water/heat. Column chromatography was carried out on columns packed with silica gel (60N spherical neutral size 40-50 $\mu \mathrm{m}$ ) for flash column chromatography. The ${ }^{1} \mathrm{H} N M R(300 \mathrm{MHz}),{ }^{19} \mathrm{~F} N \mathrm{NR}(282 \mathrm{MHz})$, and ${ }^{13} \mathrm{C}$ NMR (126 MHz) spectra for solution in $\mathrm{CDCl}_{3}, \mathrm{CD}_{3} \mathrm{OD}$ were recorded on Varian Mercury 300, and Bruker Avance 500 NMR spectrometers. 
Chemical shifts $(\delta)$ are expressed in ppm downfield from TMS $(\delta=0.00)$ or $\mathrm{C}_{6} \mathrm{~F}_{6}\left[\delta=-162.2\left(\mathrm{CDCl}_{3}\right)\right]$ as an internal standard. Mass spectra were recorded on a SHIMAZU LCMS-2020 (ESI-MS). High resolution mass spectrometric measurements were recorded on a Waters Synapt G2 HDMS (ESI-MS), a Waters GCT premier (EI-MS). Solvents $\mathrm{CH}_{3} \mathrm{CN}, \mathrm{CH}_{2} \mathrm{Cl}_{2}$, DMF were dried and distilled before use. 2-Diazo-1-phenyl-2-((trifluoromethyl)-sulfonyl)ethan1-one (3) [12c] and (Z)- $\alpha$-bromonitrostyrenes 7a-e [14] were prepared according to the known procedures.

Typical procedure for the synthesis of 5-nitro-4-aryl-pyrazole triflon (6) and 5-bromo-4-aryl-pyrazole triflon (8) from 3 and (Z)-1-(2-bromo-2-nitrovinyl) benzene (7) (Procedure A)

To a stirred solution of (Z)- $\alpha$-bromonitrostyrenes 7 (0.1 mmol, 1.0 equiv) and 2-diazo-1-phenyl-2((trifluoromethyl)-sulfonyl)ethan-1-one (3) $(0.15 \mathrm{mmol}, 1.5$ equiv) in dry $\mathrm{MeOH}(1.0 \mathrm{~mL}), \mathrm{NaOMe}$ (10.0 equiv) was added at $0{ }^{\circ} \mathrm{C}$ and the resulting mixture was stirred for 20-25 minutes under Ar atmosphere (progress of the reaction was monitored by $T L C)$. After completion, the reaction mixture was concentrated under reduced pressure. Water was added and neutralized the solution with $5 \% \mathrm{HCl}$ and extracted with ethyl acetate. The combined organic phase was washed with brine and dried over $\mathrm{Na}_{2} \mathrm{SO}_{4}$, and the mixture was evaporated in vacuo. The crude product was purified by flash column chromatography $(\mathrm{DCM} / \mathrm{MeOH}=10: 1)$ to obtain the desired substituted 5-nitro-4-phenyl-3-((trifluoromethyl)sulfonyl)-1H-pyrazole 6 and 5-bromo-4-phenyl-3((trifluoromethyl)sulfonyl)-1H-pyrazole 8.

\section{5-Nitro-4-phenyl-3-((trifluoromethyl)sulfonyl)-1H-pyrazole}

(6a) and 5-Bromo-4-phenyl-3((trifluoromethyl)sulfonyl)-1H-pyrazole (8a):

(Z)-(2-Bromo-2-nitrovinyl) benzene 7a (0.1 mmol, $0.023 \mathrm{~g}, 1.0$ equiv), 3 (0.15 mmol, $0.042 \mathrm{~g}, 1.5$ equiv) and NaOMe $\left(1.0 \mathrm{mmol}, 0.054 \mathrm{~g}, 10.0\right.$ equiv) in dry $\mathrm{MeOH}(1.0 \mathrm{~mL})$ at $0{ }^{\circ} \mathrm{C}$ for $20 \mathrm{~min}$ provided the pure product $6 \mathrm{a}$ $(0.0205 \mathrm{~g}, 64 \%)$ as a pale yellow solid and $8 \mathrm{a}(0.0068 \mathrm{~g}, 19 \%)$ as a brown solid.

6a: Mp: 313-315 ${ }^{\circ} \mathrm{C}$ (methanol); ${ }^{1} \mathrm{H}$ NMR $\left(\mathrm{CDCl}_{3}, 300 \mathrm{MHz}\right)$ 8: 7.56-7.44 (m, 3H), 7.40-7.31 (m, 2H); ${ }^{19} \mathrm{~F} \mathrm{NMR}$ $\left(\mathrm{CDCl}_{3}, 282 \mathrm{MHz}\right) \delta:-77.96$ (s, 3F); ${ }^{13} \mathrm{C} \mathrm{NMR}\left(\mathrm{CD}_{3} \mathrm{OD}, 126 \mathrm{MHz}\right) \delta: 151.51,138.17,131.37,130.22,128.86,127.58$, 125.48, 120.81 (q, J = 324.8 Hz); IR (KBr): 3581, 3334, 3066, 1963, 1673, 1560, 1506, 1378, 1232, 1110, $921 \mathrm{~cm}^{-}$ 1; $\mathrm{HRMS}$ (ESI-TOF): calcd for $\mathrm{C}_{10} \mathrm{H}_{5} \mathrm{~F}_{3} \mathrm{~N}_{3} \mathrm{O}_{4} \mathrm{~S}[\mathrm{M}-\mathrm{H}]^{-}$319.9953; found 319.9981.

8a: $\mathrm{Mp}: 152-155{ }^{\circ} \mathrm{C}(\mathrm{DCM}) ;{ }^{1} \mathrm{H}$ NMR $\left(\mathrm{CDCl}_{3}, 300 \mathrm{MHz}\right)$ 8: 7.49-7.43 (m, 3H), 7.41-7.36 (m, 2H); ${ }^{19} \mathrm{~F} \mathrm{NMR} \mathrm{(CDCl}$, $282 \mathrm{MHz}$ ) $\delta:-78.30$ (s, 3F); ${ }^{13} \mathrm{C}$ NMR $\left(\mathrm{CDCl}_{3}, 126 \mathrm{MHz}\right)$ : $130.49,129.92,129.55,129.28,128.49,127.82,126.77$, 119.51 (q, $J=326.0 \mathrm{~Hz}$ ); IR (KBr): 3642, 3210, 3116, 2672, 1959, 1654, 1533, 1386, 1114, 987, $717 \mathrm{~cm}^{-1}$; HRMS (ESI-TOF): calcd for $\mathrm{C}_{10} \mathrm{H}_{6} \mathrm{~F}_{3} \mathrm{~N}_{2} \mathrm{O}_{2} \mathrm{SBrNa}[\mathrm{M}+\mathrm{Na}]^{+}$376.9183; found 376.9177.

5-Nitro-4-(p-tolyl)-3-((trifluoromethyl)sulfonyl)-1H-pyrazole $\quad(6 b) \quad$ and $\quad 5-B r o m o-4-(p$-tolyl)-3((trifluoromethyl)sulfonyl)-1H-pyrazole (8b):

(Z)-1-(2-Bromo-2-nitrovinyl)-4-methylbenzene 7b (0.3 mmol, 0.0726 g, 1.0 equiv), 3 (0.45 mmol, 0.125 g, 1.5 equiv) and $\mathrm{NaOMe}\left(3.0 \mathrm{mmol}, 0.162 \mathrm{~g}, 10.0\right.$ equiv) in dry $\mathrm{MeOH}(3.0 \mathrm{~mL})$ at $0{ }^{\circ} \mathrm{C}$ for $25 \mathrm{~min}$ provided the pure product $\mathbf{6 b}(0.0677 \mathrm{~g}, 67 \%)$ as a pale yellow solid and $\mathbf{8 b}(0.0251 \mathrm{~g}, 23 \%)$ as a brown solid. 
6b: Mp: 305-308 ${ }^{\circ} \mathrm{C}$ (methanol); ${ }^{1} \mathrm{H}$ NMR (CDCl, $\left.300 \mathrm{MHz}\right)$ 8: 7.34-7.15 (m, 4H), $2.43(\mathrm{~s}, 3 \mathrm{H}) ;{ }^{19} \mathrm{~F} \mathrm{NMR}\left(\mathrm{CDCl}_{3}\right.$,

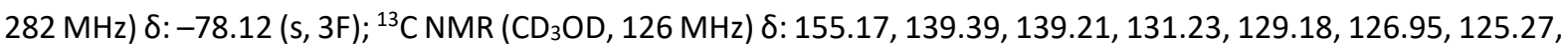
121.20 ( $\mathrm{q}, J=325.0 \mathrm{~Hz}$ ), 21.37; IR (KBr): 3596, 3210, 3035, 1720, 1670, 1529, 1371, 1224, 1110, $995 \mathrm{~cm}^{-1}$; HRMS (ESI-TOF): calcd for $\mathrm{C}_{11} \mathrm{H}_{7} \mathrm{~F}_{3} \mathrm{~N}_{3} \mathrm{O}_{4} \mathrm{~S}[\mathrm{M}-\mathrm{H}]^{-} 334.0109$, found 334.0108 .

8b: Mp: 133-134 ${ }^{\circ} \mathrm{C}(\mathrm{DCM}) ;{ }^{1} \mathrm{H}$ NMR $\left(\mathrm{CDCl}_{3}, 300 \mathrm{MHz}\right)$ 8: 7.29-7.25 (m, 4H), $2.41(\mathrm{~s}, 3 \mathrm{H}) ;{ }^{19} \mathrm{~F} \mathrm{NMR}\left(\mathrm{CDCl}_{3}, 282\right.$ $\mathrm{MHz}) \delta:-78.33$ (s, 3F); ${ }^{13} \mathrm{C} \mathrm{NMR}\left(\mathrm{CDCl}_{3}, 126 \mathrm{MHz}\right) \delta: 139.55,130.33,129.72,129.23,127.77,125.33,123.79$, $119.53(\mathrm{q}, J=325.7 \mathrm{~Hz}), 21.56$; IR (KBr): 3612, 3237, 3097, 2350, 1901, 1662, 1498, 1382, 1106, 975, $717 \mathrm{~cm}^{-1}$; HRMS (ESI-TOF): calcd for $\mathrm{C}_{11} \mathrm{H}_{8} \mathrm{~F}_{3} \mathrm{~N}_{2} \mathrm{O}_{2} \mathrm{SBrNa}[\mathrm{M}+\mathrm{Na}]^{+} 390.9340$, found 390.9333 .

4-(4-Chlorophenyl)-5-nitro-3-((trifluoromethyl)sulfonyl)-1H-pyrazole (6c) and 5-Bromo-4-(4-chlorophenyl)-3((trifluoromethyl)sulfonyl)-1H-pyrazole (8c):

(Z)-1-(2-Bromo-2-nitrovinyl)-4-chlorobenzene 7c (0.3 mmol, $0.0787 \mathrm{~g}, 1.0$ equiv), 3 ( $0.45 \mathrm{mmol}, 0.125 \mathrm{~g}$, 1.5 equiv) and $\mathrm{NaOMe}\left(3.0 \mathrm{mmol}, 0.162 \mathrm{~g}, 10.0\right.$ equiv) in dry $\mathrm{MeOH}(3.0 \mathrm{~mL})$ at $0{ }^{\circ} \mathrm{C}$ for $20 \mathrm{~min}$ provided the pure product $6 \mathrm{c}(0.0825 \mathrm{~g}, 77 \%)$ as a brownish yellow solid and $8 \mathrm{c}(0.0177 \mathrm{~g}, 15 \%)$ as a brown solid.

6c: Mp: $271-273{ }^{\circ} \mathrm{C}$ (methanol); ${ }^{1} \mathrm{H} \mathrm{NMR}\left(\mathrm{CDCl}_{3}, 300 \mathrm{MHz}\right) \delta: 7.38(\mathrm{~d}, J=8.1 \mathrm{~Hz}, 2 \mathrm{H}), 7.16(\mathrm{~d}, J=8.2 \mathrm{~Hz}, 2 \mathrm{H}), 3.04$ (br s, $1 \mathrm{H}) ;{ }^{19} \mathrm{~F} \mathrm{NMR}\left(\mathrm{CDCl}_{3}, 282 \mathrm{MHz}\right) \delta:-78.47$ (s, 3F); ${ }^{13} \mathrm{C} \mathrm{NMR}\left(\mathrm{CD}_{3} \mathrm{OD}, 126 \mathrm{MHz}\right) \delta: 156.09,139.82,135.04$, 132.99, 129.66, 128.60, 123.65, 121.26 (q, J = 325.2 Hz); IR (KBr): 3507, 3241, 3073, 2609, 1897, 1635, 1529, $1367,1220,1106,848 \mathrm{~cm}^{-1}$; HRMS (ESI-TOF): calcd for $\mathrm{C}_{10} \mathrm{H}_{4} \mathrm{~F}_{3} \mathrm{~N}_{3} \mathrm{O}_{4} \mathrm{SCl}[\mathrm{M}-\mathrm{H}]^{-} 353.9563$; found 353.9581.

8c: Mp: $136-138^{\circ} \mathrm{C}(\mathrm{DCM}) ;{ }^{1} \mathrm{H}$ NMR (CDCl, $\left.300 \mathrm{MHz}\right) \delta: 7.45(\mathrm{~d}, J=8.4 \mathrm{~Hz}, 2 \mathrm{H}), 7.33(\mathrm{~d}, J=8.4 \mathrm{~Hz}, 2 \mathrm{H}) ;{ }^{19} \mathrm{~F}$ NMR ( $\left.\mathrm{CDCl}_{3}, 282 \mathrm{MHz}\right) \delta:-78.32$ (s, 3F); $\left.{ }^{13} \mathrm{C} \mathrm{NMR} \mathrm{(CDCl} 3,126 \mathrm{MHz}\right)$ 8: 135.73, 133.76, 131.71, 130.22, 128.75, 126.43, $125.12,119.34$ (q, $J=325.9 \mathrm{~Hz}$ ); IR (KBr): 3619, 3131, 2923, 2327, 1540, 1459, 1390, 1110, 987, 829, $717 \mathrm{~cm}^{-1}$; HRMS (ESI-TOF): calcd for $\mathrm{C}_{10} \mathrm{H}_{4} \mathrm{~F}_{3} \mathrm{~N}_{2} \mathrm{O}_{2} \mathrm{SClBr}[\mathrm{M}-\mathrm{H}]^{-} 386.8817$; found 386.8824 .

4-(3-Bromophenyl)-5-nitro-3-((trifluoromethyl)sulfonyl)-1H-pyrazole (6d) and 5-Bromo-4-(3-bromophenyl)-3((trifluoromethyl)sulfonyl)-1H-pyrazole (8d):

(Z)-1-Bromo-3-(2-bromo-2-nitrovinyl)benzene 7d (0.3 mmol, $0.092 \mathrm{~g}, 1.0$ equiv), 3 ( $0.45 \mathrm{mmol}, 0.125 \mathrm{~g}$, 1.5 equiv) and $\mathrm{NaOMe}\left(3.0 \mathrm{mmol}, 0.162 \mathrm{~g}, 10.0\right.$ equiv) in dry $\mathrm{MeOH}(3.0 \mathrm{~mL})$ at $0{ }^{\circ} \mathrm{C}$ for $20 \mathrm{~min}$ provided the pure product $6 \mathbf{d}(0.0917 \mathrm{~g}, 76 \%)$ as a brownish yellow solid and $8 \mathbf{d}(0.024 \mathrm{~g}, 18 \%)$ as a brown solid.

6d: Mp: 266-268 ${ }^{\circ} \mathrm{C}$ (methanol); ${ }^{1} \mathrm{H}$ NMR (CDCl $\left.3,300 \mathrm{MHz}\right) \delta: 7.59$ (d, J = 7.5 Hz, 1H), 7.43 (s, 1H), 7.36-7.26 (m, 1H), 7.20 (d, $J=7.4 \mathrm{~Hz}, 1 \mathrm{H}$ ); $\left.{ }^{19} \mathrm{~F} \mathrm{NMR} \mathrm{(CDCl} 3,282 \mathrm{MHz}\right) \delta:-78.54(\mathrm{~s}, 3 \mathrm{~F}) ;{ }^{13} \mathrm{C}$ NMR (CD $\left.3 \mathrm{OD}, 126 \mathrm{MHz}\right) \delta: 156.12$, $139.87,134.13,133.27,131.95,130.31,130.17,123.17,122.15,121.26$ (q, $J=325.3 \mathrm{~Hz}$ ); IR (KBr): 3569, 3226, $3085,2613,1743,1552,1521,1378,1205,1106,840,624 \mathrm{~cm}^{-1}$. HRMS (ESI-TOF): calcd for $\mathrm{C}_{10} \mathrm{H}_{4} \mathrm{~F}_{3} \mathrm{~N}_{3} \mathrm{O}_{4} \mathrm{SBr}$ [MH] $]^{-}$397.9058; found 397.9055 . 
8d: Mp: $115-116{ }^{\circ} \mathrm{C}(\mathrm{DCM}) ;{ }^{1} \mathrm{H}$ NMR $\left(\mathrm{CDCl}_{3}, 300 \mathrm{MHz}\right)$ 8: 7.61 (d, J = 5.9 Hz, 1H), $7.54(\mathrm{~s}, 1 \mathrm{H}), 7.40-7.31(\mathrm{~m}, 2 \mathrm{H})$; ${ }^{19} \mathrm{~F} \mathrm{NMR}\left(\mathrm{CDCl}_{3}, 282 \mathrm{MHz}\right) \delta:-78.27$ (s, 3F); ${ }^{13} \mathrm{C}$ NMR (CD $\left.{ }_{3} \mathrm{OD}, 126 \mathrm{MHz}\right)$ 8: 134.51, 134.04, 133.04, 131.41, 130.96, 130.67, 129.47, 126.43, 122.86, 120.90 (q, J = 325.3 Hz); IR (KBr): 3667, 3139, 2938, 2373, 1556, 1459, 1375, 1106, 987, 871, 790, $655 \mathrm{~cm}^{-1}$; HRMS (ESI-TOF): calcd for $\mathrm{C}_{10} \mathrm{H}_{4} \mathrm{~F}_{3} \mathrm{~N}_{2} \mathrm{O}_{2} \mathrm{SBr}_{2}[\mathrm{M}-\mathrm{H}]^{-} 430.8312$; found 430.8305 .

4-(4-Fluorophenyl)-5-nitro-3-((trifluoromethyl)sulfonyl)-1H-pyrazole (6e) and 5-Bromo-4-(4-fluorophenyl)-3((trifluoromethyl)sulfonyl)-1H-pyrazole (8e):

(Z)-1-(2-Bromo-2-nitrovinyl)-4-fluorobenzene 7e (0.3 mmol, 0.0738 g, 1.0 equiv), 3 (0.45 mmol, $0.125 \mathrm{~g}, 1.5$ equiv) and $\mathrm{NaOMe}\left(3.0 \mathrm{mmol}, 0.162 \mathrm{~g}, 10.0\right.$ equiv) in dry $\mathrm{MeOH}(3.0 \mathrm{~mL})$ at $0{ }^{\circ} \mathrm{C}$ for 20 min provided the pure product 6 e $(0.0706 \mathrm{~g}, 69 \%)$ as a brownish yellow solid and 8 e $(0.0188 \mathrm{~g}, 17 \%)$ as a brown solid.

6e: Mp: 352-354 ${ }^{\circ} \mathrm{C}$ (methanol); ${ }^{1} \mathrm{H}$ NMR $\left(\mathrm{CDCl}_{3}, 300 \mathrm{MHz}\right) \delta: 7.26-7.23$ (m, 2H), 7.18-7.07 (m, 2H), 2.20 (br s, $1 \mathrm{H}) ;{ }^{19} \mathrm{~F} \mathrm{NMR}\left(\mathrm{CDCl}_{3}, 282 \mathrm{MHz}\right) \delta:-78.65$ (s, 3F), -111.50 (s, 1F); ${ }^{13} \mathrm{C} \mathrm{NMR}\left(\mathrm{CD}_{3} \mathrm{OD}, 126 \mathrm{MHz}\right) \delta: 162.65$ (d, J= $245.3 \mathrm{~Hz}$ ), 155.03, 138.54, 131.98 (d, J = 8.6 Hz), 125.71 (d, $J=3.8 \mathrm{~Hz}), 122.47,119.91(\mathrm{q}, J=325.2 \mathrm{~Hz}), 113.82$ (d, J = 22.0 Hz); IR (KBr): 3519, 3318, 3097, 2562, 1654, 1521, 1375, 1224, 1106, $991 \mathrm{~cm}^{-1}$; HRMS (ESI-TOF): calcd for $\mathrm{C}_{10} \mathrm{H}_{4} \mathrm{~F}_{4} \mathrm{~N}_{3} \mathrm{O}_{4} \mathrm{~S}[\mathrm{M}-\mathrm{H}]^{-} 337.9859$; found 337.9861 .

8e: $\mathrm{Mp}: 116-118{ }^{\circ} \mathrm{C}(\mathrm{DCM}) ;{ }^{1} \mathrm{H}$ NMR $\left(\mathrm{CDCl}_{3}, 300 \mathrm{MHz}\right)$ 8: 7.40-7.33 (m, 2H), 7.19-7.12 (m, 2H); ${ }^{19} \mathrm{~F} \mathrm{NMR}\left(\mathrm{CDCl}_{3}\right.$, $282 \mathrm{MHz}) \delta:-78.35$ (s, 3F), -111.67--111.86 (m, 1F); ${ }^{13} \mathrm{C} N M R\left(\mathrm{CD}_{3} \mathrm{OD}, 126 \mathrm{MHz}\right) \delta: 164.57$ (d, $\left.J=247.0 \mathrm{~Hz}\right)$, $133.89(\mathrm{~d}, J=8.7 \mathrm{~Hz}), 130.72,129.47,127.02,125.31$ (d, $J=3.6 \mathrm{~Hz}), 120.92$ (q, $J=325.1 \mathrm{~Hz}), 116.10$ (d, $J=22.1$ $\mathrm{Hz}$ ); IR (KBr): 3679, 3127, 2931, 2377, 1548, 1475, 1382, 1228, 1114, 983, 755, $640 \mathrm{~cm}^{-1}$; HRMS (ESI-TOF): calcd for $\mathrm{C}_{10} \mathrm{H}_{4} \mathrm{~F}_{4} \mathrm{~N}_{2} \mathrm{O}_{2} \mathrm{SBr}[\mathrm{M}-\mathrm{H}]^{-} 370.9113$; found 370.9113 .

\section{Synthesis of 4-(4-chlorophenyl)-1-(pyrimidin-2-yl)-3-((trifluoromethyl)sulfonyl)-1H-pyrazol-5-amine (2a)}

To a solution of 4-(4-chlorophenyl)-5-nitro-3-((trifluoromethyl)sulfonyl)-1H-pyrazole 6c (0.0355 g, 0.1 $\mathrm{mmol}$ ) in $\mathrm{MeOH}$ was added $\mathrm{Pd} / \mathrm{C}(0.006 \mathrm{~g})$, and the mixture was stirred at rt under $\mathrm{H}_{2}$ atmosphere (balloon) for $2.5 \mathrm{~h}$. The mixture was filtered through a pad of Celite ${ }^{\circledR}$ to give the amine 9 . The crude product was used for the next reaction without further purification.

The crude amine 9 was dissolved in DMF $(1.0 \mathrm{~mL})$. NaH (60\% w/w in mineral oil, $0.0024 \mathrm{~g}, 0.1 \mathrm{mmol})$ was added to the mixture at $0{ }^{\circ} \mathrm{C}$, and the mixture was stirred at rt for $30 \mathrm{~min}$. 2-Chloropyrimidine $(0.0115 \mathrm{~g}, 0.1$ mmol) was added to the mixture and the mixture was stirred at $100{ }^{\circ} \mathrm{C}$ for $12 \mathrm{~h} . \mathrm{H}_{2} \mathrm{O}$ was added to the mixture and the mixture was extracted with EtOAc. The combined organic phase was washed with brine and dried with $\mathrm{Na}_{2} \mathrm{SO}_{4}$, and the mixture was evaporated in vacuo. The crude product was purified with flash column chromatography (eluted with $\mathrm{CH}_{2} \mathrm{Cl}_{2}: \mathrm{MeOH}=20: 1$, the silica gel was neutralized with $1 \%$ ammonia solution in DCM prior to using) to give the 5-amino- $N$-pyrimidinyl-pyrazole triflone $2 \mathrm{a}(0.014 \mathrm{~g}, 35 \%$ yield) as a white solid.

Mp (DCM): $150-151{ }^{\circ} \mathrm{C} ;{ }^{1} \mathrm{H}$ NMR $\left(300 \mathrm{MHz}, \mathrm{CDCl}_{3}\right) \delta: 8.90$ (d, J=4.9 Hz, 2H), 7.48-7.41 (m, 3H), 7.40-7.34 (m,

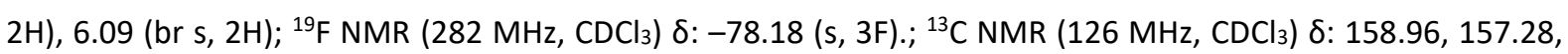


148.99, 143.03, 130.72, 129.02, 128.75, 128.20, 119.74, 119.68 (q, J = 326.3 Hz), 106.98; IR (KBr): 3411, 3255, $3060,1600,1567,1511,1428,1376,1297,1213,1120,979,838,794,701,657 \mathrm{~cm}^{-1}$; HRMS (ESI-TOF): calcd for $\mathrm{C}_{14} \mathrm{H}_{8} \mathrm{~F}_{3} \mathrm{~N}_{5} \mathrm{O}_{2} \mathrm{SCl}[\mathrm{M}-\mathrm{H}]^{-}$402.0039; found 402.0029.

\section{Acknowledgements}

This work was supported by JSPS KAKENHI grants JP $16 \mathrm{H} 01142$ (Middle Molecular Strategy) and JP16H01017 (Precisely Designed Catalysts with Customized Scaffolding), and ACT-C from the JST (JPMJCR12Z7). ET thanks the Pesticide Science Society of Japan for support.

\section{References}

1. (a) Lee, K. Y.; Kim, J. M.; Kim, J. N. Tetrahedron Lett. 2003, 44, 6737-6740. (b) Elgemeie, G. H.; Zaghary, W. A.; Amin, K. M.; Nasr, T. M. Nucleosides, Nucleotides Nucleic Acids 2005, 24, 1227-1247. (c) McDonald, E.; Jones, K.; Brough, P. A.; Drysdale, M. J.; Workman, P. Curr. Top. Med. Chem. 2006, 6, 1193-2003. (d) Lamberth, C. Heterocycles 2007, 71, 1467-1502. (e) Dolzhenko, A. V.; Dolzhenko, A. V.; Chui, W. K. Heterocycles 2008, 75, 1575-1622. (f) Fustero, S.; Román, R.; Sanz-Cervera, J.-F.; SimónFuentes, A.; Cunãt, A. C.; Villanova, S.; Murguía, M. J. Org. Chem. 2008, 73, 3523-3529. (g) Fustero, S.; Sanz-Cervera, J. F.; Simón-Fuentes, A.; Román, R.; Catalán, S.; Murguía, M. ACS Symp. Ser. 2009, 1003, 182-209.

2. (a) Kane, J. L.; Hirth, B. H.; Liang, B.; Gourlie, B. B.; Nahill, S.; Barsomian, G. Bioorg. Med. Chem. Lett. 2003, 13, 4463-4466. (b) Kumar, V.; Aggarwal, R.; Tyagi, P.; Singh, S. P. Europ. J. Med. Chem. 2005, 40, 922-927. (c) Aggarwal, R.; Kumar, V.; Tyagi, P.; Singh, S. P. Bioorg. Med. Chem. 2006, 14, 1785-1791.

3. (a) Frackenpohl, J.; Gebauer, O.; Cerezo-Galvez, S.; Es-Sayed, M.; Gorgens, U.; Franken, E.-M.; Malsam, O.; Schnatterer, S.; Arnold, C.; Lummen, P.; Schwarz, H.-G.; Hense, S.; Werner, S. PCT Int. Appl. WO 2008077483 A1, 2008. (b) Kudo, N.; Furuta, S.; Taniguchi, M.; Endo, T.; Sato, K. Chem. Pharm. Bull. 1999, $47,857-868$.

4. (a) Banks, B. J. Eur. Pat. Appl. EP 846686 A1, 1998. (b) Banks, B. J. U.S. Patent 6,069,157A, 2000.

5. Meegalla, S. K.; Doller, D.; Sha, D.-Y.; Soll, R.; Wisnewski, N.; Silver, G. M.; Dhanoa, D. Bioorg. Med. Chem. Lett. 2004, 14, 4949-4953.

6. (a) Food Standards Agency: Update on Fipronil in eggs, https://www.food.gov.uk/newsupdates/news/2017/16427/update-on-fipronil-in-eggs-10-august; (b) Health assessment of individual measurements of Fipronil levels detected in foods of animal origin in Belgium, DOI 10.17590/20170802140011

7. Schwarz, H.-G.; Schenke, T.; Hense, A.; Velten, R.; Maechling, S.; Werner, S.; Franken, E.-M.; Gorgens, U.; Lummen, P.; E.-Kiintscher, U.; Voerste, A. PCT Int. Appl. WO 2009156090 A2, 2009.

8. (a) Filler, R.; Kobayashi, Y. Biomedical Aspects of Fluorine Chemistry; Elsevier Biomedical Press and Kodansha Ltd.: Amsterdam, Tokyo, 1982. (b) Filler, R.; Kobayashi, Y.; Yagupolskii, L. M. (Ed) 
Organofluorine Compounds in Medicinal Chemistry and Biomedical Applications; Elsevier: Amsterdam, New York, 1993.

9. Xu, X.-H.; Shibata, N. J. Synth. Org. Chem. Jpn. 2013, 71, 1195-1201.

10. (a) Xu, X.-H.; Taniguchi, M.; Azuma, A.; Liu, G.; Tokunaga, E.; Shibata, N. Org. Lett. 2013, 15, 686-689. (b) Xu, X.-H.; Taniguchi, M.; Wang, X.; Tokunaga, E.; Ozawa, T.; Masuda, H.; Shibata, N. Angew. Chem., Int. Ed. 2013, 52, 12628-12631. (c) Kawai, H.; Sugita, Y.; Tokunaga, E.; Sato, H.; Shiro, M.; Shibata, N. ChemistryOpen 2014, 3, 14-18. (d) Das, P.; Shibata, N. J. Org. Chem. 2017, 82, 11915-11924.

11. (a) Shibata, N.; Ishimaru, T.; Nakamura, S.; Toru, T. J. Fluorine Chem. 2007, 128, 469-483. (b) Shibata, N.; Mizuta, S.; Kawai, H. Tetrahedron: Asymmetry 2008, 19, 2633-2644. (c) Shibata, N.; Matsnev, A.; Cahard, D. Beilstein J. Org. Chem. 2010, 6, No. 65. (d) Kawai, H.; Shibata, N. Chem. Rec. 2014, 14, 1024-1040. (e) Shibata, N. Bull. Chem. Soc. Jpn. 2016, 89, 1307-1320.

12. (a) Huang, Z.; Okuyama, K.; Wang, C.; Tokunaga, E.; Li, X.; Shibata, N. ChemistryOpen 2016, 5, $188-191$. (b) Huang, Z.; Jia, S.; Wang, C.; Tokunaga, E.; Sumii, Y.; Shibata, N. J. Fluorine Chem. 2017, 198, 61-66. (c) Huang, Z.; Wang, C.; Tokunaga, E.; Sumii, Y.; Shibata, N. Org. Lett. 2015, 17, 5610-5613. (d) Das, P.; Gondo, S.; Tokunaga, E.; Sumi, Y.; Shibata, N. Org. Lett. 2018, 20, 558-561.

13. (a) Muruganantham, R.; Mobin, S. M.; Namboothiri, I. N. N. Org. Lett. 2007, 9, 1125-1128. (b) Xie, J.W.; Wang, Z.; Yang, W.-J.; Konga, L.-C.; Xu, D.-C. Org. Biomol. Chem. 2009, 7, 4352-4354. (c) Muruganantham, R.; Namboothiri, I. J. Org. Chem. 2010, 75, 2197-2205.

14. Huang, K.; Ma, Q.; Shen, X.; Gong, L.; Meggers, E. Asian J. Org. Chem. 2016, 5, 1198-1203. 


\title{
Supporting Information
}

\section{Synthesis of 5-nitro-pyrazole triflones via [3+2] cycloaddition reaction and its application for potential insecticide}

\author{
Pulakesh Das, ${ }^{1}$ Hiroto Uno, ${ }^{1}$ Etsuko Tokunaga,${ }^{1}$ Yuji Sumii ${ }^{1}$ and Norio Shibata ${ }^{1,2 *}$ \\ ${ }^{1}$ Department of Nanopharmaceutical Sciences, and Department of Life Science and Applied Chemistry, \\ Nagoya Institute of Technology, Gokiso, Showa-ku, Nagoya 466-8555, Japan \\ E-mail: nozshiba@nitech.ac.jp \\ ${ }^{2}$ Institute of Advanced Fluorine-Containing Materials, Zhejiang Normal University, 688 Yingbin Avenue, \\ 321004 Jinhua, China
}

Table of contents

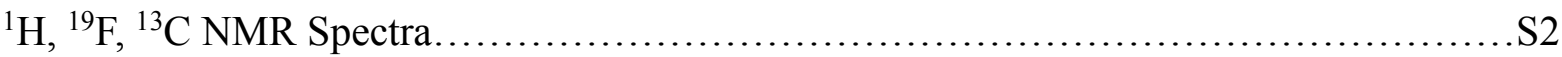



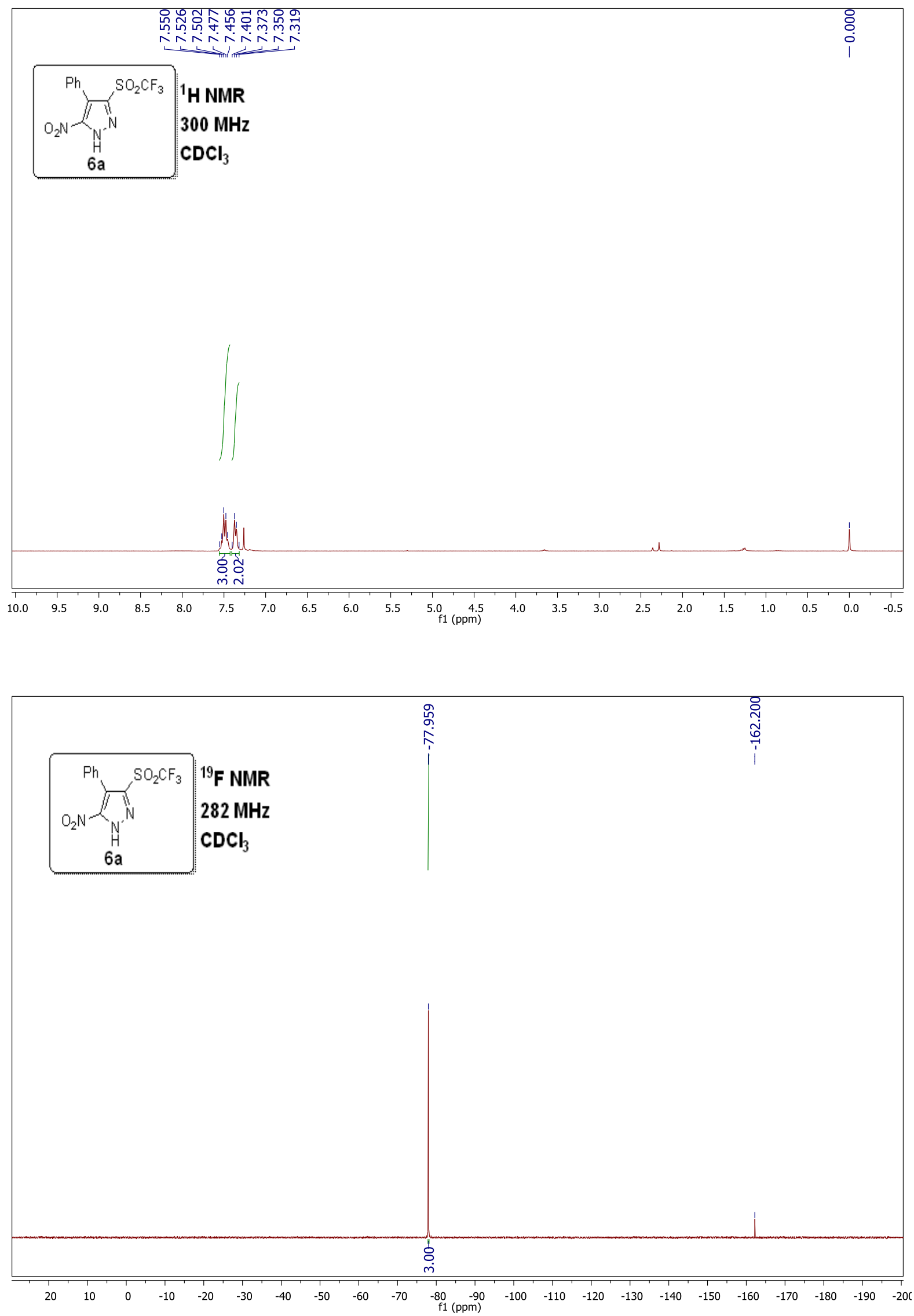

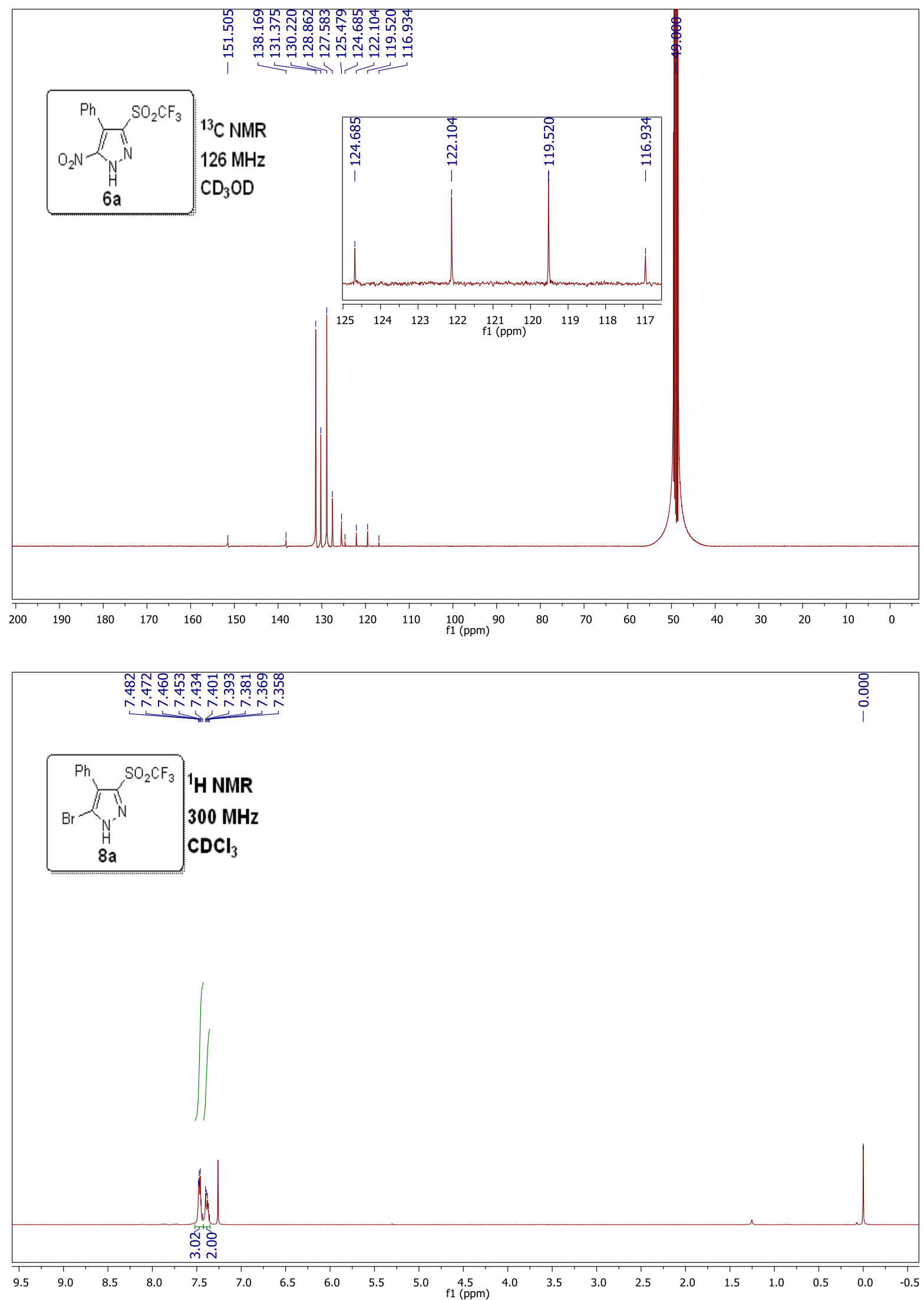

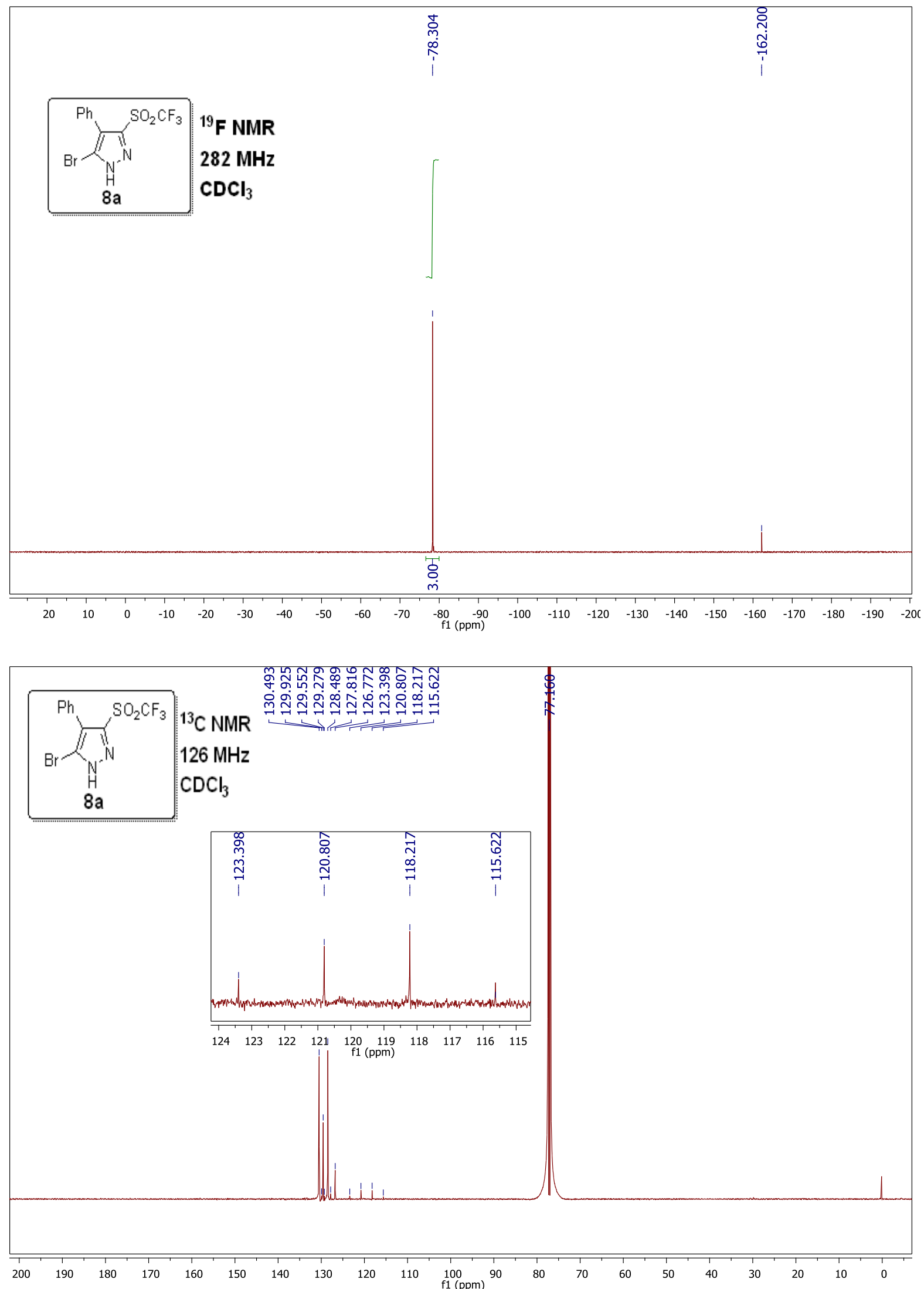

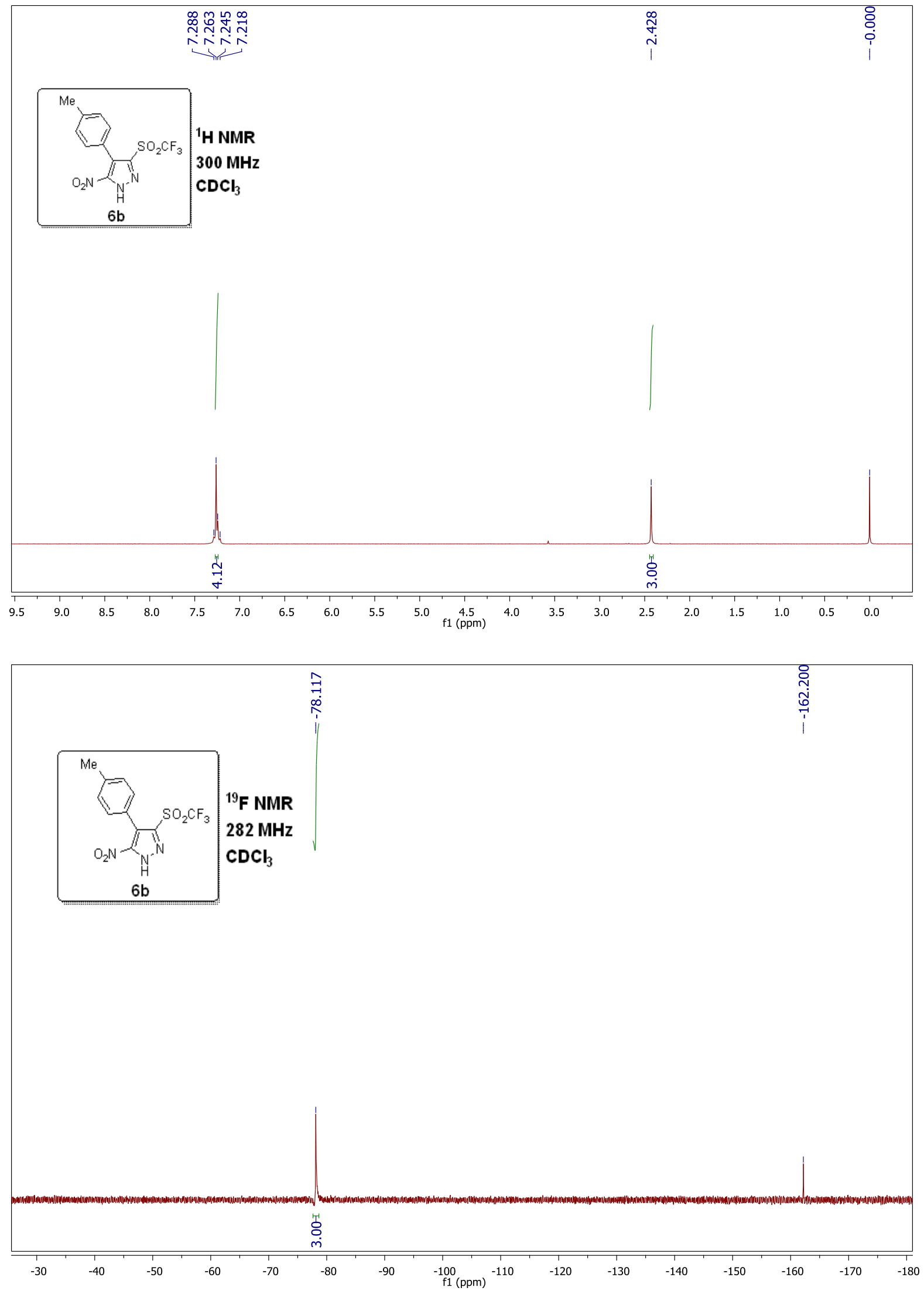

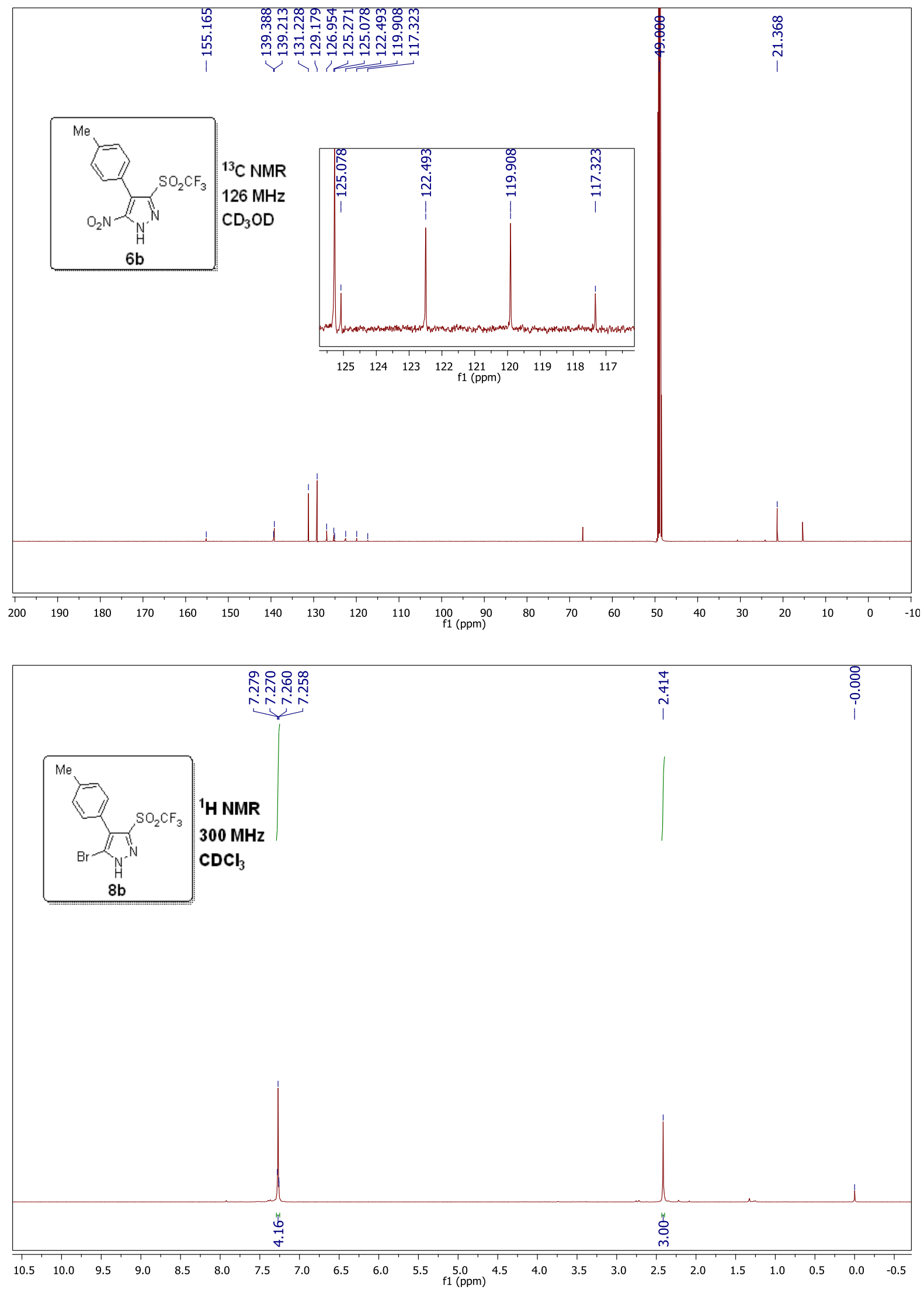

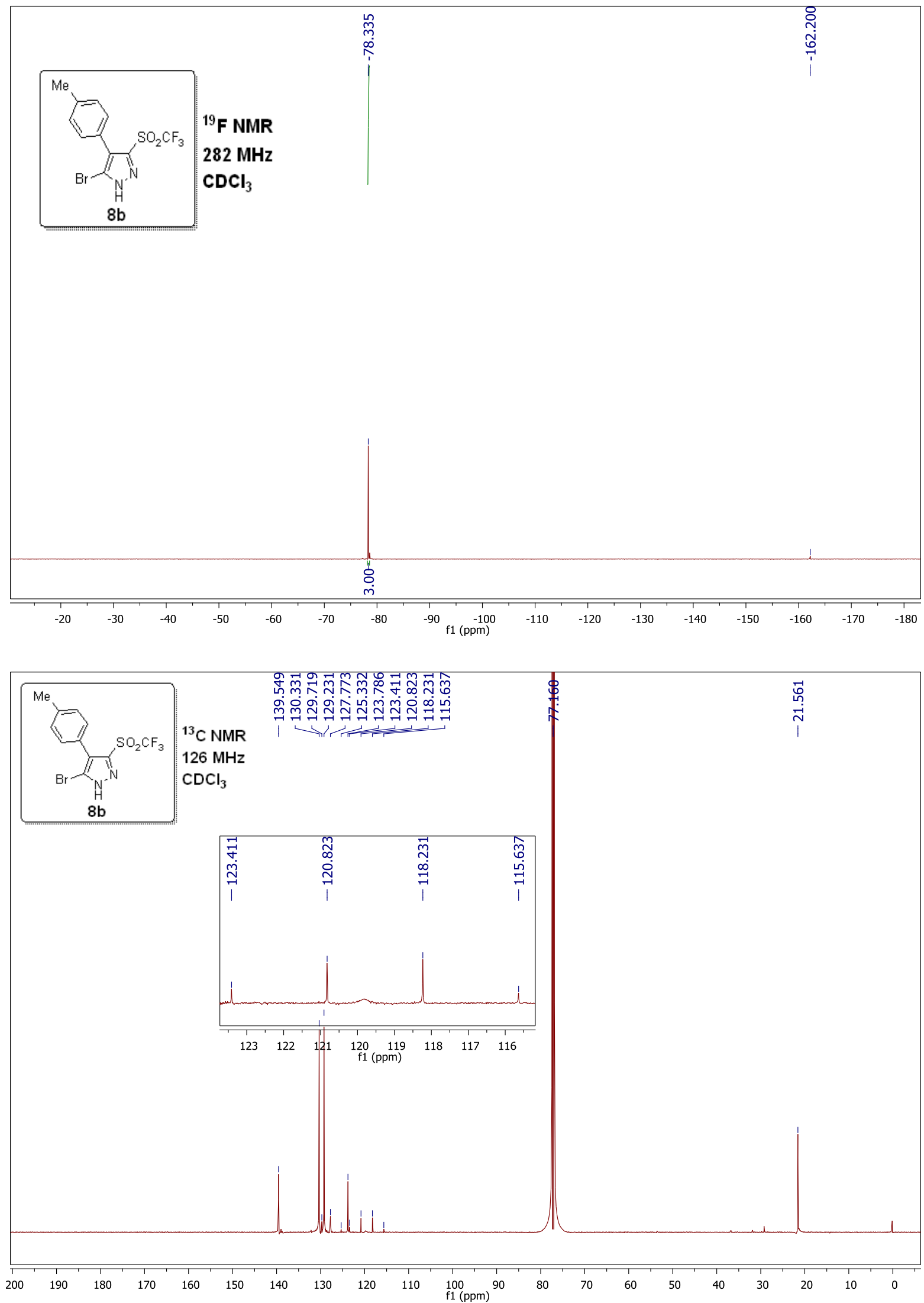

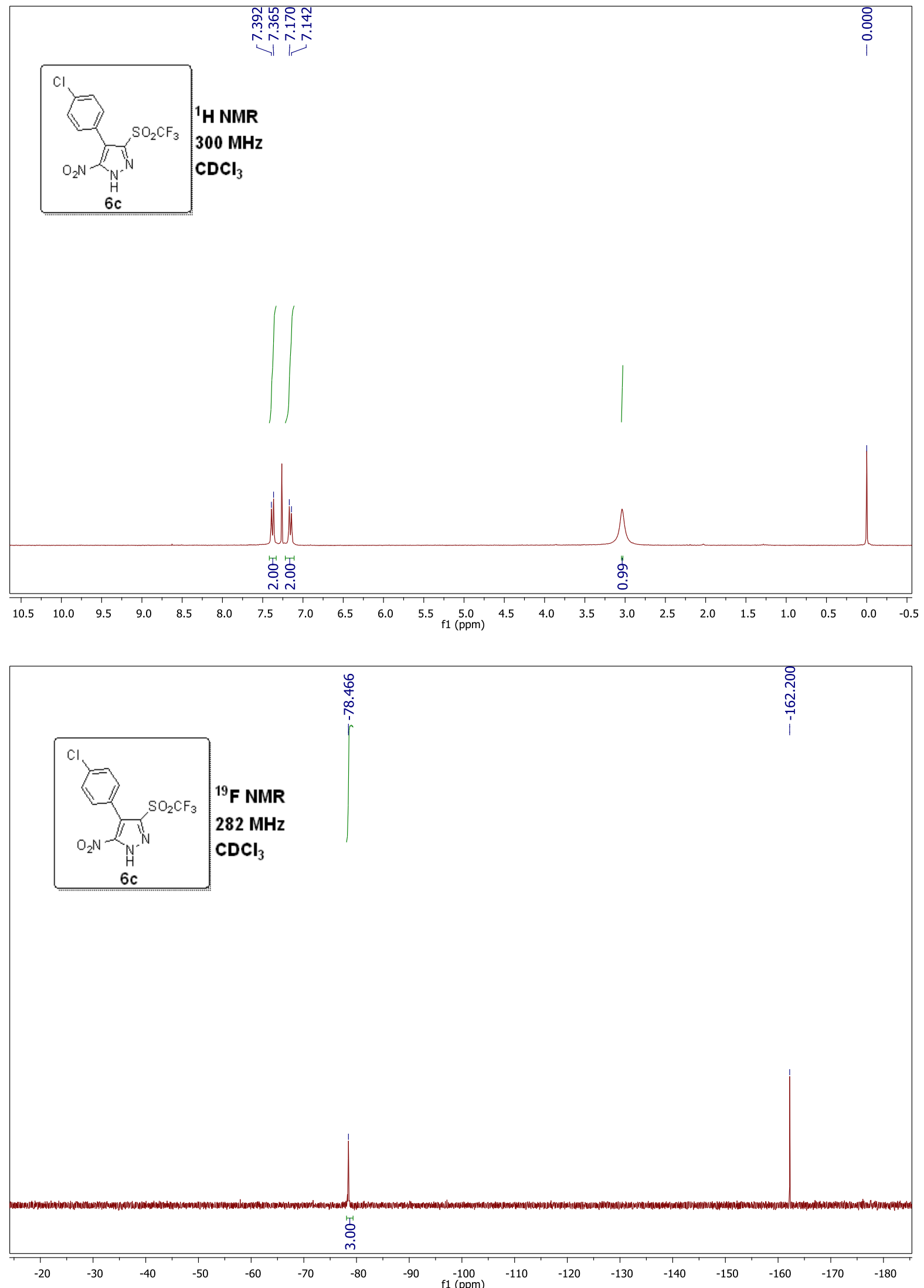

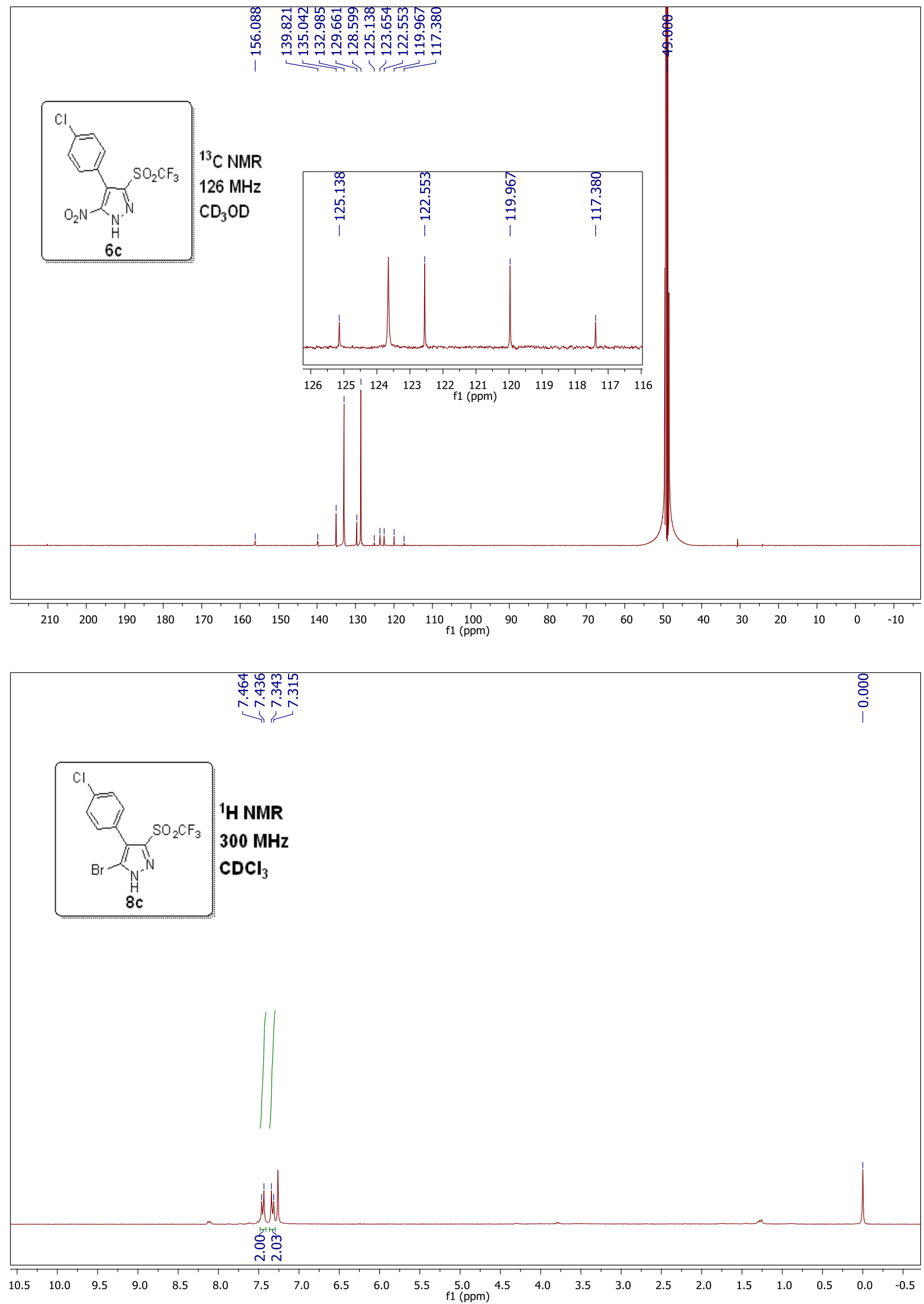

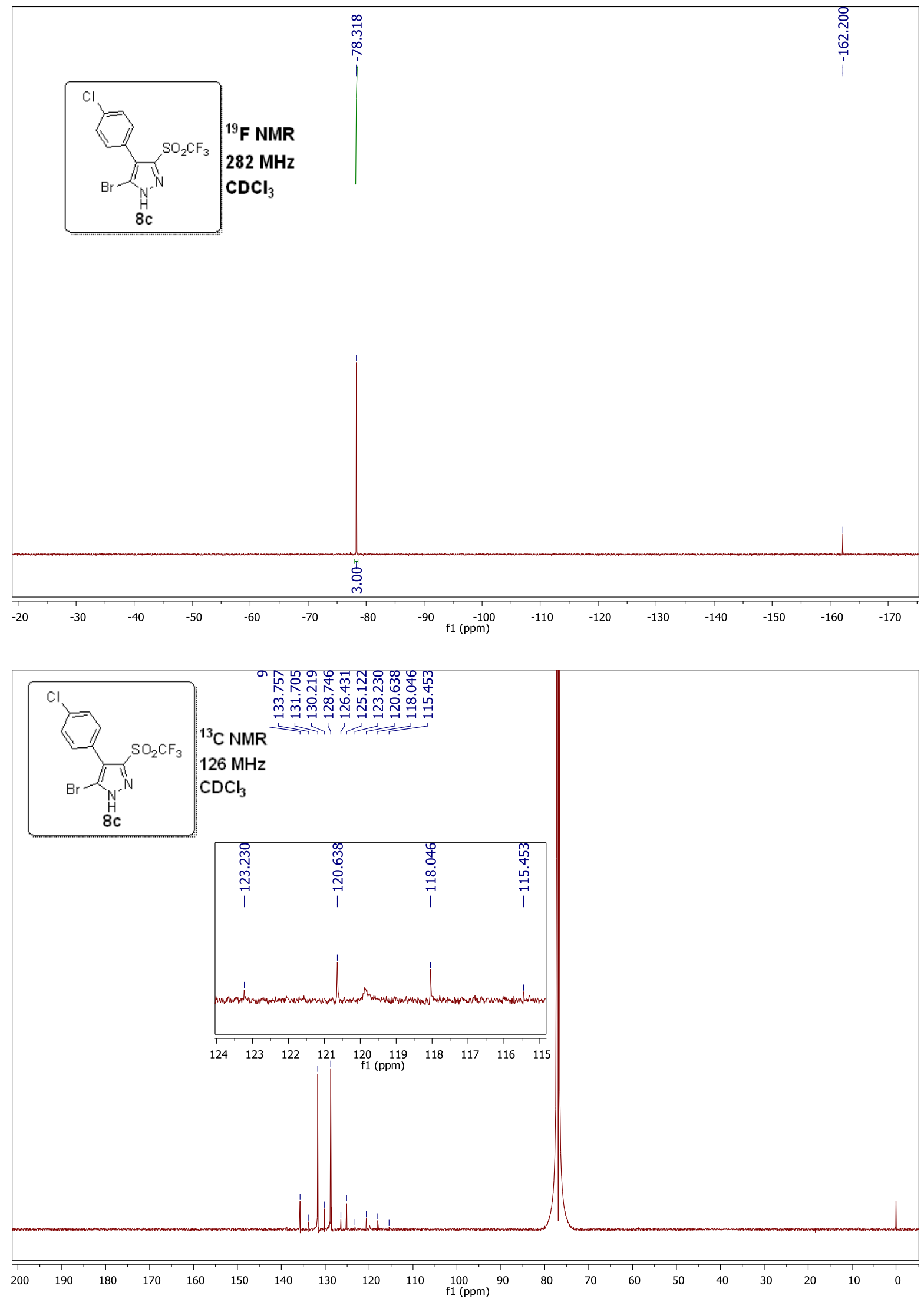

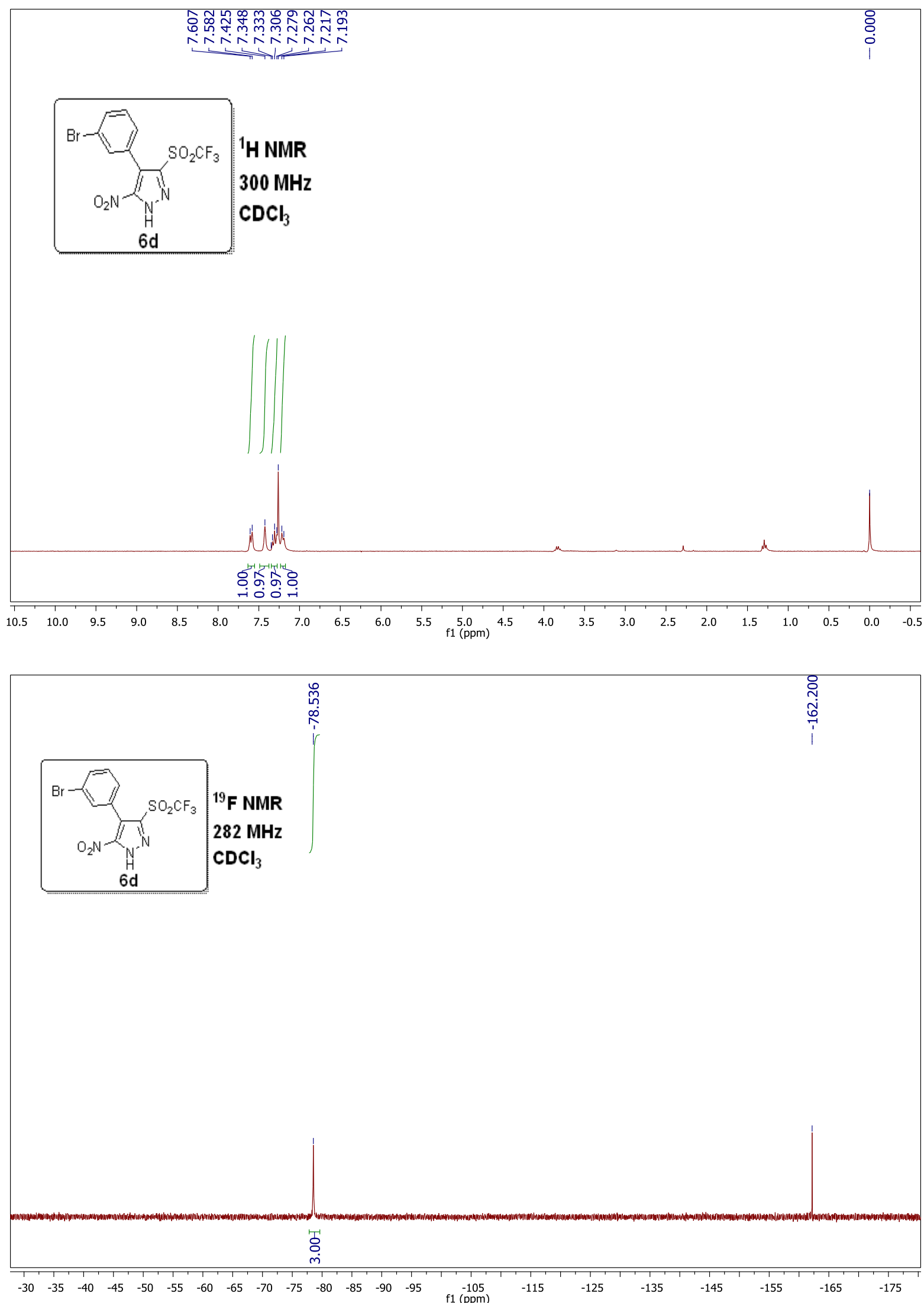

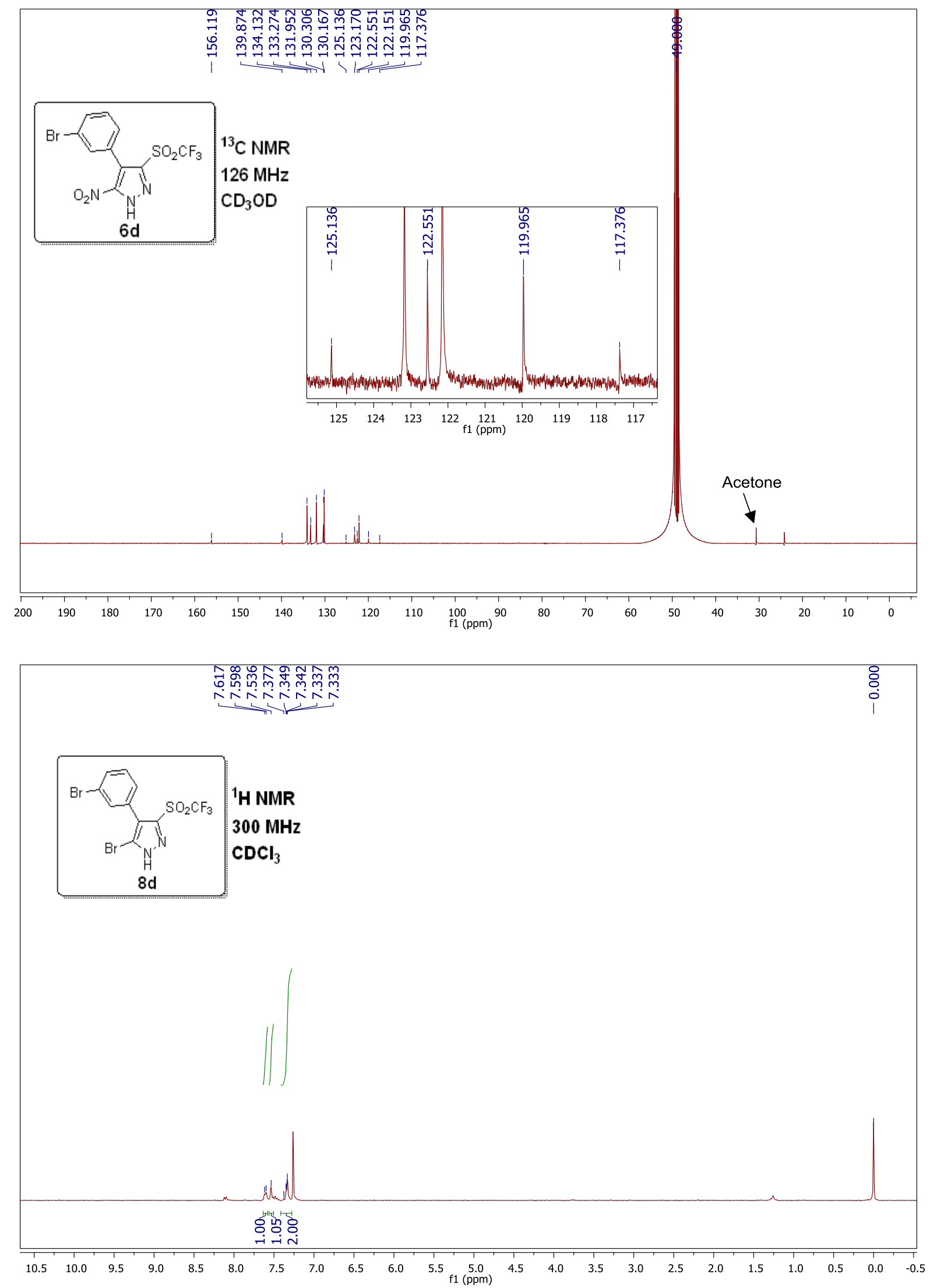

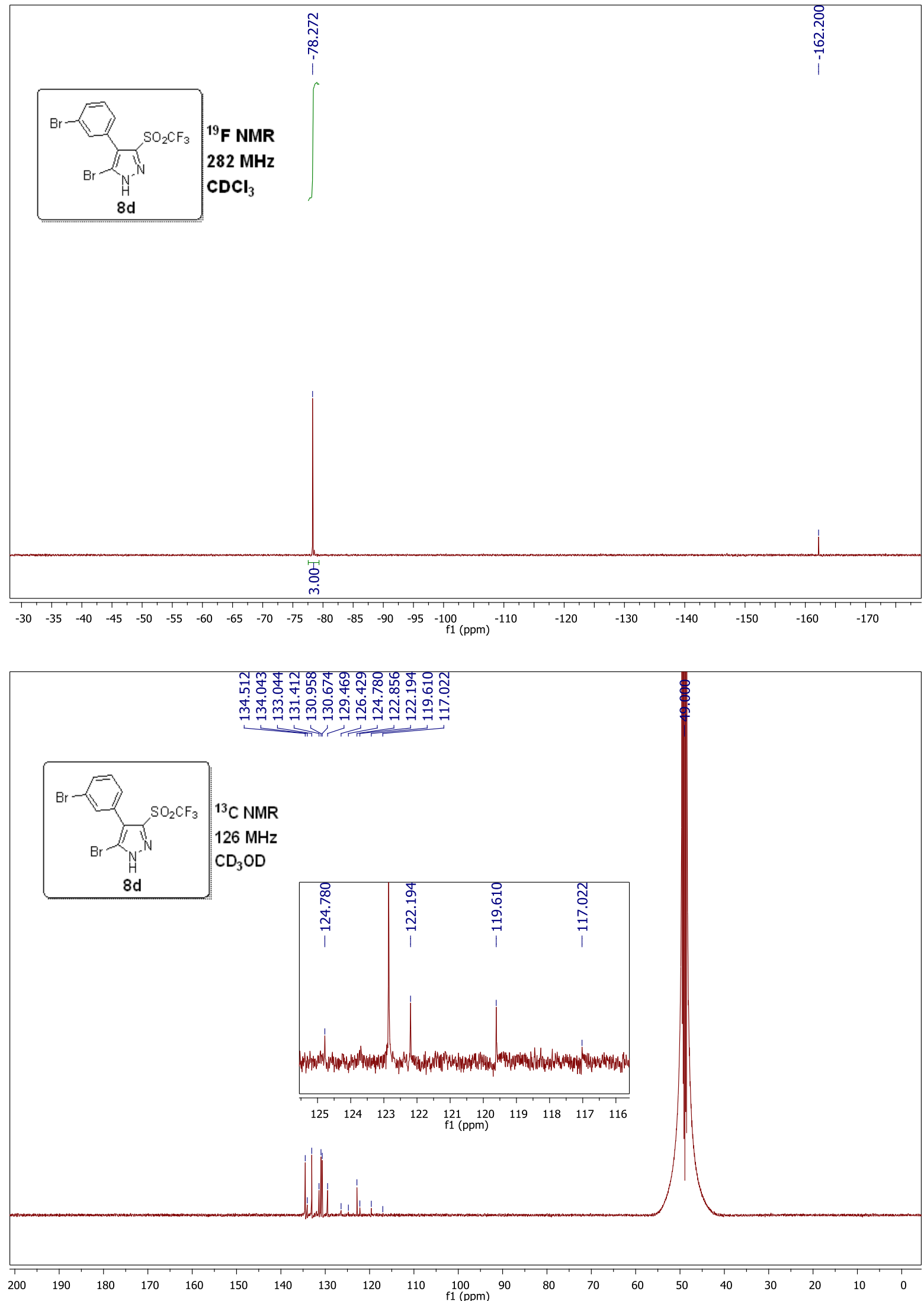

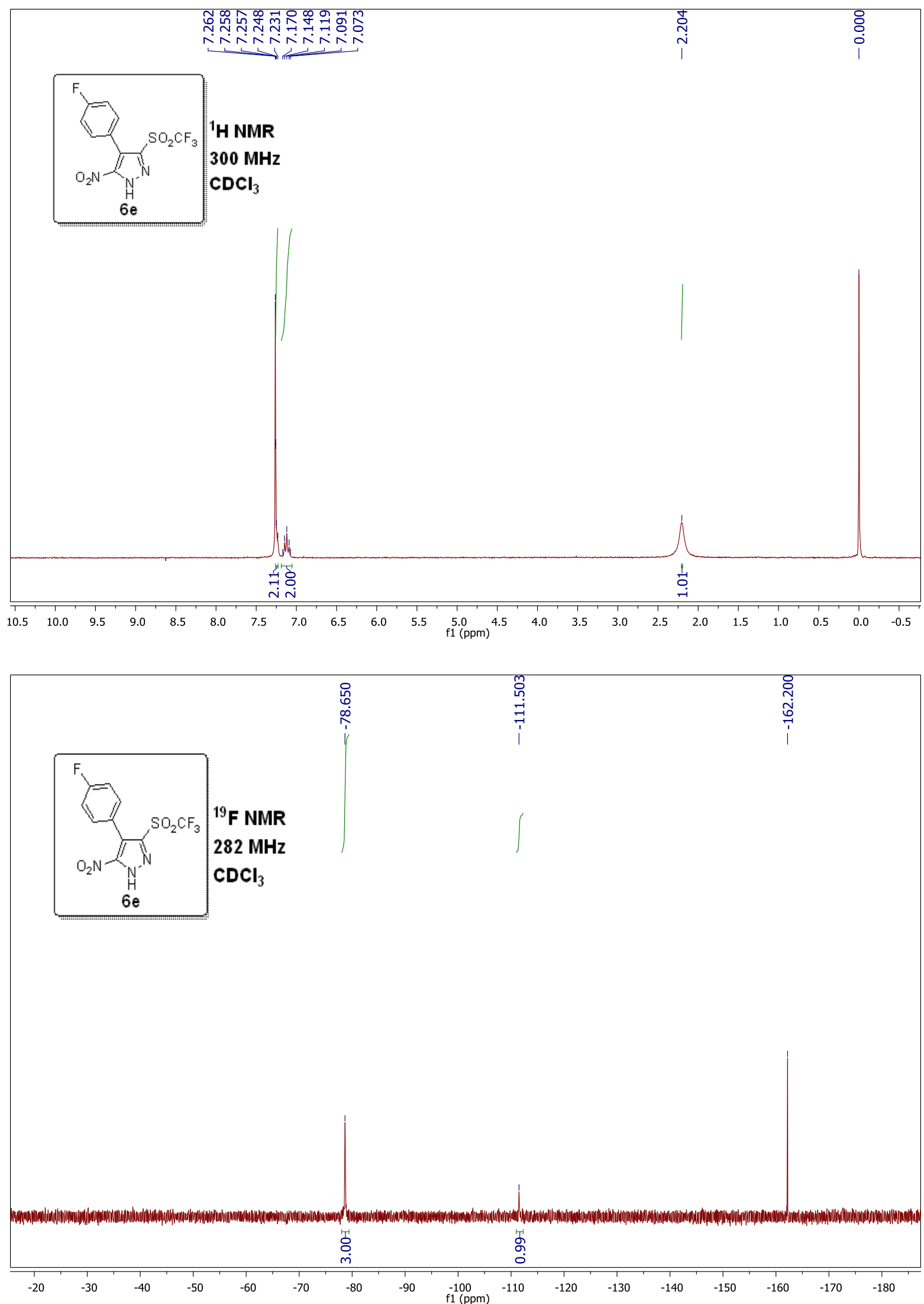

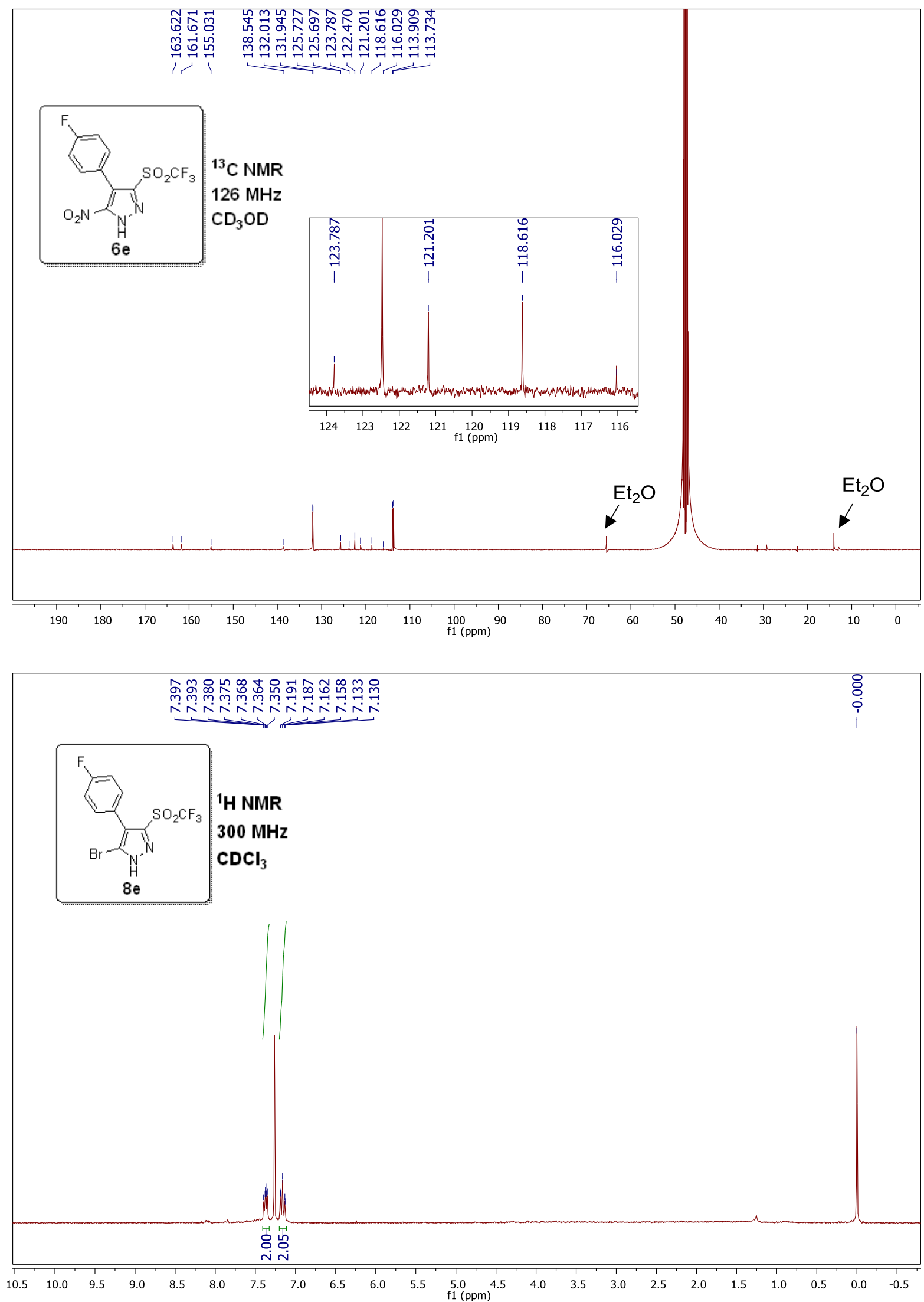

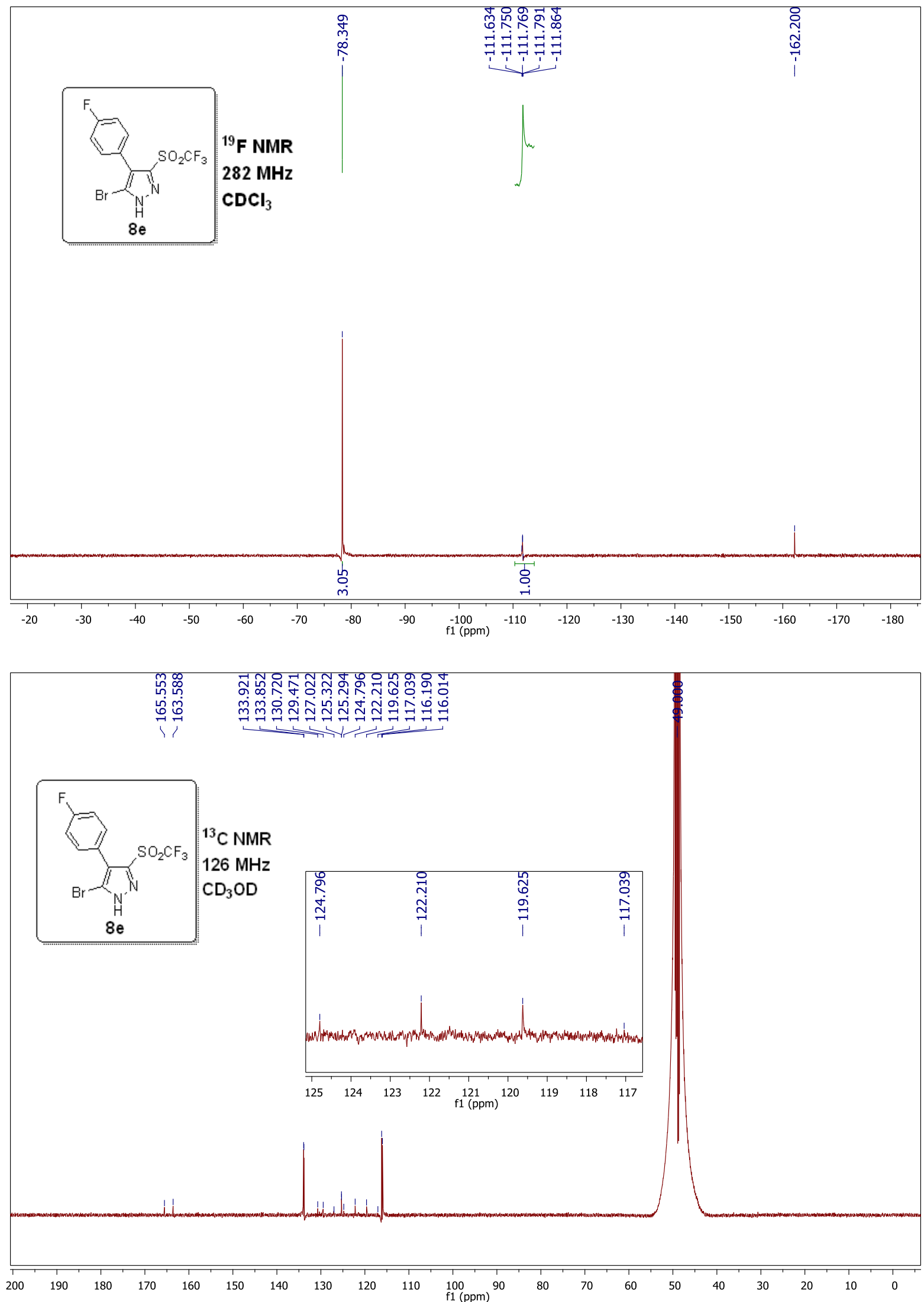

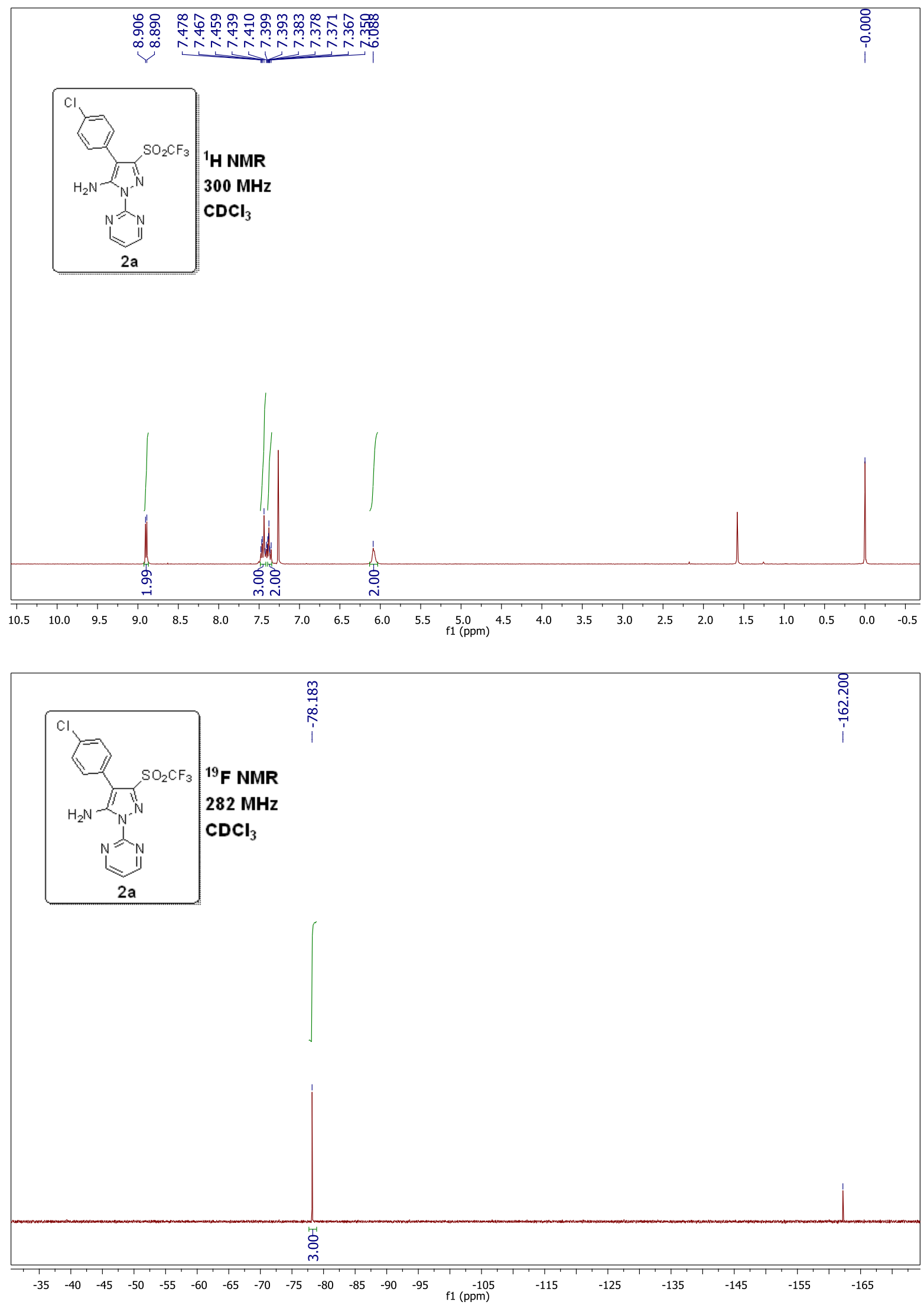


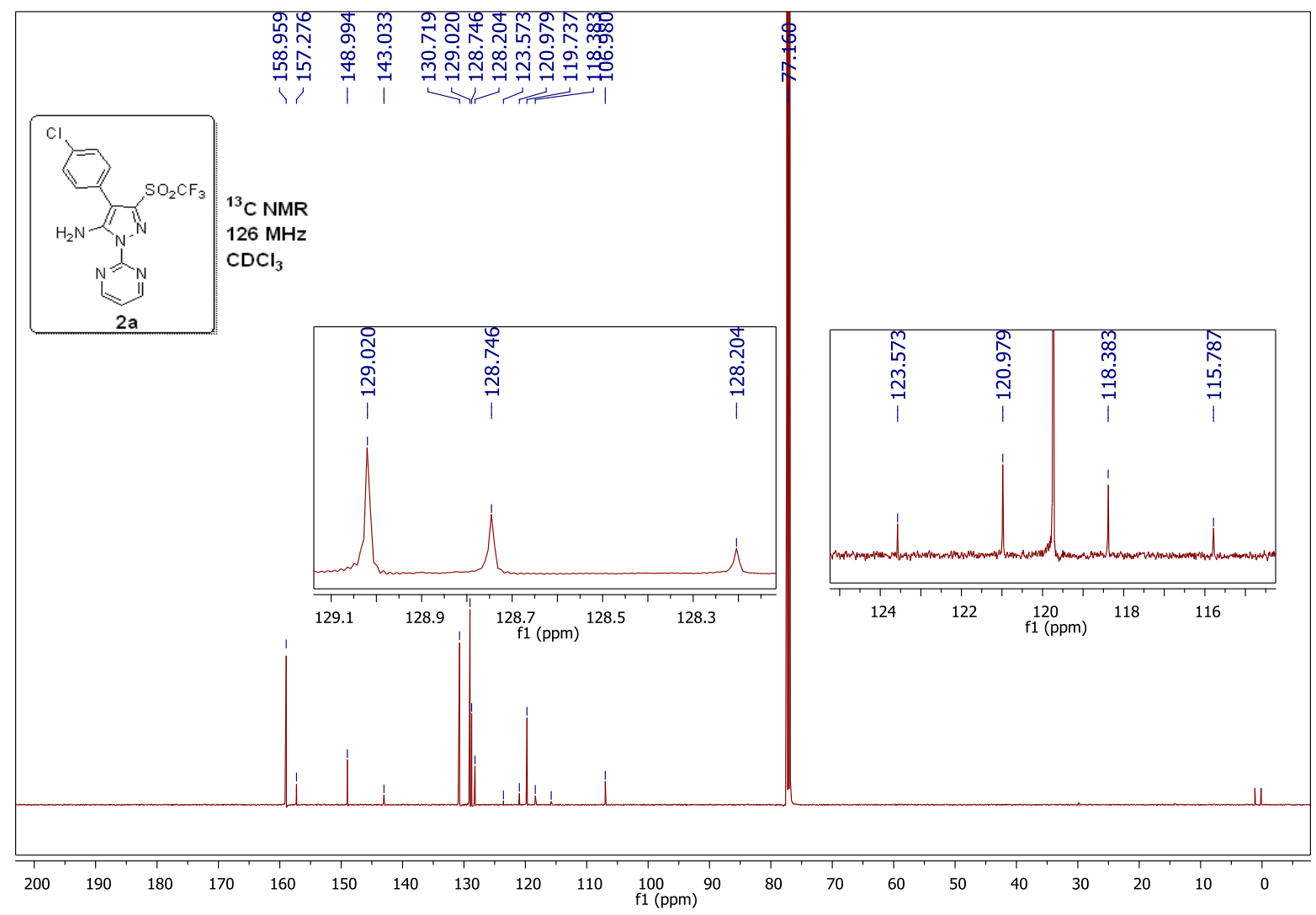

